\title{
Triple collinear splitting functions at NLO for scattering processes with photons
}

\author{
Germán F.R. Sborlini, ${ }^{a, b}$ Daniel de Florian ${ }^{a}$ and Germán Rodrigo ${ }^{b}$ \\ ${ }^{a}$ Departamento de Física and IFIBA, FCEyN, Universidad de Buenos Aires, \\ (1428) Pabellón 1 Ciudad Universitaria, Capital Federal, Argentina \\ ${ }^{b}$ Instituto de Física Corpuscular, Universitat de València, \\ Consejo Superior de Investigaciones Científicas, \\ Parc Cientific, E-46980 Paterna, Valencia, Spain \\ E-mail: gfsborlini@df.uba.ar, deflo@df.uba.ar, german.rodrigo@csic.es
}

ABSTRACT: We present splitting functions in the triple collinear limit at next-to-leading order. The computation was performed in the context of massless QCD+QED, considering only processes which include at least one photon. Through the comparison of the IR divergent structure of splitting amplitudes with the expected known behavior, we were able to check our results. Besides that we implemented some consistency checks based on symmetry arguments and cross-checked the results among them. Studying photon-started processes, we obtained very compact results.

Keywords: NLO Computations

ArXiv EPRINT: 1408.4821 


\section{Contents}

1 Introduction 1

2 Multiple-collinear limit and IR divergent behavior $\quad 2$

3 Splitting functions with photons $\quad \mathbf{5}$

3.1 Review of double-collinear results 5

$\begin{array}{ll}3.2 \quad q \rightarrow q \gamma \gamma & 7\end{array}$

$\begin{array}{ll}3.3 q \rightarrow q g \gamma & 10\end{array}$

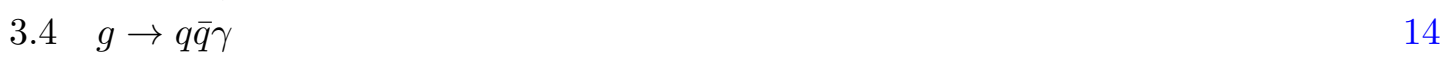

4 Processes started by photons 18

$\begin{array}{lll}4.1 & \gamma \rightarrow q \bar{q} \gamma & 18\end{array}$

$\begin{array}{ll}4.2 \gamma \rightarrow q \bar{q} g & 19\end{array}$

4.3 Further discussions on splittings started by photons 22

5 Conclusions $\quad 25$

\section{Introduction}

Studying the collinear limit of scattering amplitudes is a key point for improving theoretical computations. This is a requirement to compare theoretical predictions with the highlyaccurate results provided by the LHC and other experiments at colliders. In this context, understanding the singular behavior of scattering amplitudes in the multiple-collinear limit is necessary to obtain higher order QCD corrections to several processes.

It is a well known fact that, in certain general kinematical configurations, strict collinear factorization is fulfilled [2]. ${ }^{1}$ These factorization properties establish that it is possible to isolate the singular behavior of scattering amplitudes into universal factors called splitting amplitudes [3, 4]. At the level of squared amplitudes, the Altarelli-Parisi (AP) kernels or splitting functions control the infrared (IR) singular behavior. Moreover, splitting functions govern the evolution of parton distributions functions (PDF) and fragmentation functions (FF) through the DGLAP equation [5]. Also, they are the main ingredient in parton-shower generators and they are crucial for subtraction methods [6] to compute physical cross sections at higher orders in perturbation theory.

Double-collinear splitting amplitudes and squared amplitudes have been computed at one-loop [7-12] and two-loop level [13-17]. Splitting kernels beyond the double collinear limit, however, are known at the tree-level only [18-23], although some specific results for the triple collinear limit of one-loop amplitudes are already available [24].

\footnotetext{
${ }^{1}$ See also [1] and references therein.
} 
In this article, we present explicit results for unpolarized squared splitting amplitudes at next-to-leading order (NLO) in QCD for triple collinear processes involving at least one photon. In the context of dimensional regularization (DREG) [25, 26], we work in conventional dimensional regularization (CDR) to make explicit the IR divergent structure, with $d=4-2 \epsilon$ the number of space-time dimensions. The corresponding squared splitting amplitudes in other schemes can be obtained by computing scalar-gluon contributions or by using transition rules if we are only interested in the $\mathcal{O}\left(\epsilon^{0}\right)$ terms. Transition rules for scattering amplitudes were previously discussed in the literature [27-29], while we treated this topic for the double-collinear limit in ref. [30]. We will not enter into this discussion in this paper.

The outline of the paper is the following. In section 2 we describe the multiplecollinear kinematics and introduce some relevant properties and notation. After some brief comments about QCD with photons, we review the double-collinear splitting functions for processes with photons (both at the amplitude and squared-amplitude level). Then, we present explicit results for NLO unpolarized splitting kernels with one and two photons in section 3. To be more specific, we compute $q \rightarrow q \gamma \gamma, q \rightarrow q g \gamma$ and $g \rightarrow q \bar{q} \gamma$ at NLO in QCD. In section 4 we show results for triple splitting kernels involving one photon which splits into three partons: $\gamma \rightarrow q \bar{q} \gamma$ and $\gamma \rightarrow q \bar{q} g$. In this section, we also present a discussion about the functional structure of these objects, using the photon-started splitting processes to handle more compact expressions. Finally we present the conclusions and perspectives in section 5 .

\section{Multiple-collinear limit and IR divergent behavior}

To explore the multiple collinear limit, we consider an $n$-particle process in which a subset of $m$ particles become simultaneously collinear. We label the collinear particles with $i \in C=\{1,2, \ldots, m\}$. If $p_{i}^{\mu}$ is the four-momentum associated with particle $i$, and assuming $p_{i}^{2}=0$ (massless on-shell partons), then the subenergies $s_{i j}=2 p_{i} \cdot p_{j}$ and $s_{i, j}=\left(p_{i}+p_{i+1}+\ldots+p_{j}\right)^{2}=p_{i, j}^{2}$, with $i$ and $j \in C$, are the only dimensional relevant quantities involved in the collinear limit. We define a pair of light-like vectors $\left(\widetilde{P}^{2}=0\right.$, $\left.n^{2}=0\right)$, such that

$$
\widetilde{P}^{\mu}=p_{1, m}^{\mu}-\frac{s_{1, m}}{2 n \cdot \widetilde{P}} n^{\mu}
$$

approaches the collinear direction in the multiparton collinear limit, and $n^{\mu}$ parametrizes how the collinear limit is approached, with $n \cdot \widetilde{P}=n \cdot p_{1, m}$. The longitudinal-momentum fractions $z_{i}$ of the collinear partons are

$$
z_{i}=\frac{n \cdot p_{i}}{n \cdot \widetilde{P}}, \quad i \in C
$$

and fulfil the constraint $\sum_{i \in C} z_{i}=1$. We work in the physical-gauge where the factorization properties are explicit, with

$$
d_{\mu \nu}(k, n)=-\eta_{\mu \nu}+\frac{k_{\mu} n_{\nu}+n_{\mu} k_{\nu}}{n \cdot k}
$$

the physical polarization tensor of a gauge vector boson (gluon or photon) with momentum $k$. The auxiliary gauge vector $n$ in eq. (2.3) is taken identical to the light-like vector $n$ introduced in eq. (2.1). 
On the other hand, let's recall some universal properties of the collinear limit of scattering amplitudes. Strict collinear factorization is fulfilled to all orders in the time-like region (i.e. where $s_{i j}>0$ for all $i, j \in C$ ). For simplicity, we limit ourselves to this region for the rest of the paper and leave the computation of factorization breaking effects in the space-like region $[2,31]$ for a future publication. When particles 1 to $m$ become collinear, the one-loop matrix element $\mathcal{M}^{(1)}$ factorizes according to

$$
\begin{aligned}
\left|\mathcal{M}^{(1)}\left(p_{1}, \ldots, p_{n}\right)\right\rangle \simeq & \boldsymbol{S p}_{a_{1} \ldots a_{m}}^{(1)}\left(p_{1}, \ldots, p_{m} ; \widetilde{P}\right)\left|\mathcal{M}^{(0)}\left(\widetilde{P}, p_{m+1}, \ldots, p_{n}\right)\right\rangle \\
& +\boldsymbol{S p}_{a_{1} \ldots a_{m}}^{(0)}\left(p_{1}, \ldots, p_{m} ; \widetilde{P}\right)\left|\mathcal{M}^{(1)}\left(\widetilde{P}, p_{m+1}, \ldots, p_{n}\right)\right\rangle
\end{aligned}
$$

which is valid for the most singular terms in the collinear limit. The factors $\boldsymbol{S p}^{(0)}$ and $\boldsymbol{S} \boldsymbol{p}^{(1)}$, also called splitting matrices, encode the singular behavior in the multiple collinear limit at tree-level and one-loop, respectively. As discussed in ref. [2], they turn out to be process-independent in the time-like region as they depend on the momenta and quantum numbers (flavour, spin, and color) of the collinear partons only. Centering in $\boldsymbol{S p}^{(1)}$, it can be expressed as

$$
\boldsymbol{S p}_{a_{1} \ldots a_{m}}^{(1)}=\boldsymbol{S p}_{a_{1} \ldots a_{m}}^{(1) \text { div. }}+\boldsymbol{S} \boldsymbol{p}_{a_{1} \ldots a_{m}}^{(1) \text { fin. }}
$$

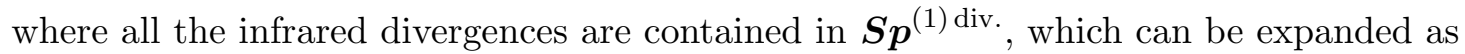

$$
\boldsymbol{S} \boldsymbol{p}_{a_{1} \ldots a_{m}}^{(1) \text { div. }}\left(p_{1}, \ldots, p_{m} ; \widetilde{P}\right)=\boldsymbol{I}_{a_{1} \ldots a_{m}}^{(1)}\left(p_{1}, \ldots, p_{m} ; \widetilde{P}\right) \boldsymbol{S p}_{a_{1} \ldots a_{m}}^{(0)}\left(p_{1}, \ldots, p_{m} ; \widetilde{P}\right),
$$

with

$$
\begin{aligned}
\boldsymbol{I}_{a_{1} \ldots a_{m}}^{(1)}\left(p_{1}, \ldots, p_{m} ; \widetilde{P}\right)= & c_{\Gamma} g_{\mathrm{S}}^{2}\left(\frac{-s_{1, m}-i 0}{\mu^{2}}\right)^{-\epsilon}\left\{\frac{1}{\epsilon^{2}} \sum_{i, j=1(i \neq j)}^{\bar{m}} \boldsymbol{T}_{i} \cdot \boldsymbol{T}_{j}\left(\frac{-s_{i j}-i 0}{-s_{1, m}-i 0}\right)^{-\epsilon}\right. \\
& +\frac{1}{\epsilon^{2}} \sum_{i, j=1}^{\bar{m}} \boldsymbol{T}_{i} \cdot \boldsymbol{T}_{j}\left(2-\left(z_{i}\right)^{-\epsilon}-\left(z_{j}\right)^{-\epsilon}\right) \\
& \left.-\frac{1}{\epsilon}\left(\sum_{i=1}^{\bar{m}}\left(\gamma_{i}-\epsilon \tilde{\gamma}_{i}^{\text {R.S. }}\right)-\left(\gamma_{a}-\epsilon \tilde{\gamma}_{a}^{\text {R.S }}\right)-\frac{\tilde{m}-2}{2}\left(\beta_{0}-\epsilon \tilde{\beta}_{0}^{\text {R.S }}\right)\right)\right\},(2.7
\end{aligned}
$$

where the color matrix of the collinear particle with momentum $p_{i}$ is denoted by $\boldsymbol{T}_{i}, \bar{m}$ counts the number of collinear final state QCD partons and $\tilde{m}$ refers to the total number of QCD partons in the splitting process. We also define

$$
c_{\Gamma}=\frac{\Gamma(1+\epsilon) \Gamma(1-\epsilon)^{2}}{(4 \pi)^{2-\epsilon} \Gamma(1-2 \epsilon)},
$$

as the usual one-loop $d$-dimensional volume factor. We can appreciate that $\tilde{m}=\bar{m}$ in collinear splittings which are started by non-QCD partons. We order the final state particles such that $\{1, \ldots, \bar{m}\}$ are the colored ones while the remaining ones are singlets under $\mathrm{SU}\left(N_{\mathrm{C}}\right)$ transformations. The flavour coefficients $\gamma_{i}$ and $\beta_{0}$ are $\gamma_{q}=\gamma_{\bar{q}}=3 C_{F} / 2$ and $\gamma_{g}=\beta_{0} / 2=\left(11 C_{A}-2 N_{f}\right) / 6$. Up to $\mathcal{O}\left(\epsilon^{0}\right)$, the regularization scheme (RS) dependence is controlled by the coefficients $\tilde{\gamma}_{i}^{\text {R.S }}$ and $\tilde{\beta}_{0}^{\text {R.S. }}$. Explicitly, we have

$$
\tilde{\gamma}_{i}^{\text {C.D.R. }}=\tilde{\beta}_{0}^{\text {C.D.R. }}=0
$$


in conventional dimensional regularization $(\mathrm{CDR})$, while

$$
\begin{aligned}
& \tilde{\gamma}_{q}^{\text {D.R. }}=\tilde{\gamma}_{\bar{q}}^{\text {D.R }}=C_{F} / 2, \\
& \tilde{\gamma}_{g}^{\text {D.R. }}=\tilde{\beta}_{0}^{\text {D.R }} / 2=C_{A} / 6,
\end{aligned}
$$

in dimensional reduction (DR). Moreover, we consider unrenormalized quantities, so $g_{\mathrm{S}}$ is the bare QCD coupling. In the curly bracket of eq. (2.7), the contribution proportional to $\beta_{0}-\epsilon \tilde{\beta}_{0}^{\text {R.S. }}$ is of ultraviolet origin, and it will be removed by working at the level of renormalized matrix elements and splitting matrices.

The factor $\boldsymbol{I}_{a_{1} \ldots a_{m}}^{(1)}$ in eq. (2.7) is a matrix in the color space. However, since color conservation implies that the color charge of the parent parton is given by

$$
\sum_{i} \boldsymbol{T}_{i} \boldsymbol{S p}_{a_{1} \ldots a_{m}}^{(0)}=\boldsymbol{S} \boldsymbol{p}_{a_{1} \ldots a_{m}}^{(0)} \boldsymbol{T}_{a}
$$

and since we consider processes with at most $\tilde{m}=3$ QCD partons, the color algebra can be carried out in closed form and $\boldsymbol{I}_{a_{1} \ldots a_{m}}^{(1)}$ becomes proportional to the unit matrix, namely, it becomes a $c$-number that we denote $I_{a_{1} \ldots a_{m}}^{(1)}$.

The square of the splitting matrix $\boldsymbol{S} \boldsymbol{p}_{a_{1} \ldots a_{m}}$, summed over final-state colors and spins and averaged over colors and spins of the parent parton, defines the $m$-parton unpolarized splitting function $\left\langle\hat{P}_{a_{1} \ldots a_{m}}\right\rangle$, which is a generalization of the customary Altarelli-Parisi double collinear splitting function. Fixing the normalization of the tree-level splitting function by

$$
\left\langle\hat{P}_{a_{1} \cdots a_{m}}^{(0)}\right\rangle=\left(\frac{s_{1, m}}{2 \mu^{2 \epsilon}}\right)^{m-1} \overline{\left|\boldsymbol{S} \boldsymbol{p}_{a_{1} \ldots a_{m}}^{(0)}\right|^{2}},
$$

then it is possible to use eq. (2.5) and eq. (2.6) to present the NLO correction as

$$
\begin{aligned}
\left\langle\hat{P}_{a_{1} \cdots a_{m}}^{(1)}\right\rangle & =\left(\frac{s_{1, m}}{2 \mu^{2 \epsilon}}\right)^{m-1}\left(\overline{\boldsymbol{S p}_{a_{1} \ldots a_{m}}^{(1)}\left(\boldsymbol{S} \boldsymbol{p}_{a_{1} \ldots a_{m}}^{(0)}\right)^{\dagger}}+\text { h.c. }\right) \\
& =2 \operatorname{Re}\left(I_{a_{1} \cdots a_{m}}^{(1)}\left(p_{1}, \ldots, p_{m} ; \widetilde{P}\right)\right)\left\langle\hat{P}_{a_{1} \cdots a_{m}}^{(0)}\right\rangle+\left(\left\langle\hat{P}_{a_{1} \cdots a_{m}}^{(1) f i n .}\right\rangle+\text { c.c. }\right),
\end{aligned}
$$

with the aim of exposing explicitly the divergent structure of the splitting function. Then, working with the finite remainder, we classify each term according to its transcendental weight. So, the second step of the decomposition that we carried out is to express the finite contribution to the NLO unpolarized splitting kernel as

$$
\left\langle\hat{P}_{a_{1} \cdots a_{m}}^{(1) \text { fin. }}\right\rangle=c^{a_{1} \cdots a_{m}}\left[C^{(0)}+\sum_{i=1}^{2} \sum_{j} C_{j}^{(i)} F_{j}^{(i)}\left(\left\{s_{r l}, z_{k}\right\}\right)+\mathcal{O}(\epsilon)\right],
$$

where $F_{j}^{(i)}$ denotes a basis of a function's space with transcendental weight $i$ and $C_{j}^{(i)}$ are the corresponding coefficients. Here $c^{a_{1} \cdots a_{m}}$ is a normalization factor which depends on the process and includes all the couplings. 


\section{Splitting functions with photons}

In this section we present explicit results for the NLO corrections to triple collinear splitting functions involving photons. We describe the corresponding model through the Lagrangian density given by

$$
\mathcal{L}_{\mathrm{QCD}+\mathrm{QED}}=\mathcal{L}_{\mathrm{QCD}}-\mu^{\epsilon} g_{e} \sum_{q} e_{q} \delta_{i j} \bar{\Psi}_{q}^{i} \gamma^{\mu} \Psi_{q}^{j} A_{\mu}-\frac{1}{4} F^{\mu \nu} F_{\mu \nu},
$$

where $\{i, j\}$ are color indices, $g_{e}$ is the electromagnetic coupling (i.e. the absolute value of electron charge), $e_{q}$ is the charge of quark's flavour $q\left(e_{u, c, t}=2 / 3\right.$ and $\left.e_{d, s, b}=-1 / 3\right)$ and $F_{\mu \nu}=\partial_{\mu} A_{\nu}-\partial_{\nu} A_{\mu}$ is the gauge-field strength tensor for the Abelian group $\mathrm{U}(1)_{E}$. The photon-quark interaction is proportional to the identity matrix Id in the color space.

Besides that, the computation is performed in the time-like region. With this kinematic choice, we can ensure strict factorization properties (as claimed in ref. [2]). Centering in the triple collinear limit and using the notation previously introduced, we have $m=3$ but $\bar{m}$ is not fixed. Since we are interested in QCD corrections, we consider first splitting processes where the parent parton is a QCD parton. For $\bar{m}=1$, we have the splitting $a \rightarrow a \gamma \gamma$ with $a$ a quark or gluon. Since $\boldsymbol{S p}_{g \gamma \gamma}^{(0)}=0$, the one-loop correction $\boldsymbol{S p}_{g \gamma \gamma}^{(1)}$ is ultraviolet finite and the only allowed unpolarized splitting function at NLO with $\bar{m}=1$ is $\left\langle\hat{P}_{q \gamma \gamma}^{(1)}\right\rangle$. When $\bar{m}=2$, with a photon in the final state, there are more possibilities. The relevant splittings functions are $\left\langle\hat{P}_{q g \gamma}\right\rangle,\left\langle\hat{P}_{q \bar{q} \gamma}\right\rangle$, and $\left\langle\hat{P}_{g g \gamma}\right\rangle$ where $\boldsymbol{S p}_{g g \gamma}^{(0)}=0$. Finally, splitting processes initiated by photons will be discussed in section 4 .

As mentioned in the introduction, the computation is performed using CDR scheme in order to simplify the treatment of intermediate expressions. It is possible to obtain DR scheme results up to $\mathcal{O}\left(\epsilon^{0}\right)$ by replacing the flavour coefficients and $\tilde{\beta}_{0}^{\text {R.S. }}$ in eq. $(2.7)$. Also, we could obtain the exact expressions in other schemes by computing the corresponding scalar-gluon contributions [30], although in this work we are only interested in $\mathcal{O}\left(\epsilon^{0}\right)$ corrections.

We start showing results for the $q \rightarrow q \gamma \gamma$ splitting function at NLO. Then, we express $q \rightarrow q g \gamma$ in terms of the previous one, in order to simplify the results. Finally, we compute $g \rightarrow q \bar{q} \gamma$ using the techniques mentioned above.

\subsection{Review of double-collinear results}

Before starting with the triple-collinear splitting functions, let's show some results for the double-collinear limit of scattering amplitudes with photons. This topic has been analysed in ref. [30] where we also discussed some technical details related with DREG schemes. In the context of QCD+QED, we have only two double-collinear splitting processes: $q \rightarrow q \gamma$ and $\gamma \rightarrow q \bar{q}$.

The corresponding Feynman diagrams required to perform the computation are shown in figure 1. Using them, we obtained both splitting amplitudes and unpolarized splitting functions up to NLO accuracy. The results for the LO contributions are

$$
\boldsymbol{S p}_{q_{1} \gamma_{2}}^{(0)}=\frac{g_{e} e_{q} \mu^{\epsilon}}{s_{12}} \mathbf{I d} \bar{u}\left(p_{1}\right) \notin\left(p_{2}\right) u(\widetilde{P}),
$$



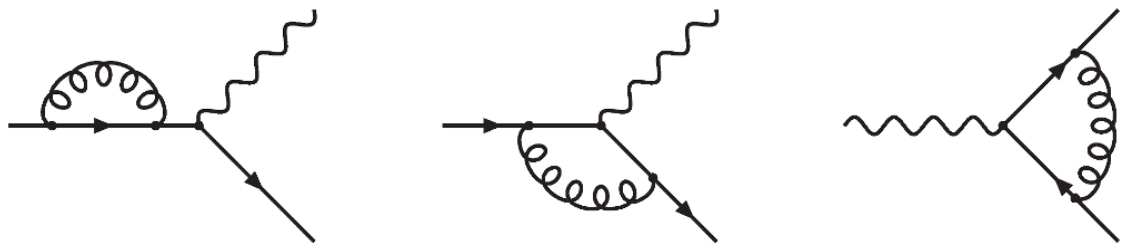

Figure 1. Feynman diagrams contributing to the splitting amplitudes of $q \rightarrow q \gamma$ and $\gamma \rightarrow q \bar{q}$.

$$
\boldsymbol{S p}_{\gamma \rightarrow q_{1} \bar{q}_{2}}^{(0)}=\frac{g_{e} e_{q} \mu^{\epsilon}}{s_{12}} \mathbf{I d} \bar{u}\left(p_{1}\right) \notin(\widetilde{P}) v\left(p_{2}\right),
$$

and

$$
\begin{aligned}
\boldsymbol{S p}_{q_{1} \gamma_{2}}^{(1)}= & c_{\Gamma} g_{\mathrm{S}}^{2} C_{F}\left(\frac{-s_{12}-\imath 0}{\mu^{2}}\right)^{-\epsilon}\left[\frac{2}{\epsilon^{2}}\left(1-{ }_{2} F_{1}\left(1,-\epsilon ; 1-\epsilon ; \frac{z_{1}-1}{z_{1}}\right)+\frac{\epsilon^{2}}{2(2 \epsilon-1)}\right) \boldsymbol{S p}_{q_{1} \gamma_{2}}^{(0)}\right. \\
& \left.-\frac{g_{e} e_{q} \mu^{\epsilon}}{s_{12} n \cdot \widetilde{P}(2 \epsilon-1)} \mathbf{I d} \bar{u}\left(p_{1}\right) \hbar u(\widetilde{P}) p_{1} \cdot \epsilon\left(p_{2}\right)\right] \\
\boldsymbol{S p}_{\gamma \rightarrow q_{1} \bar{q}_{2}}^{(1)}= & c_{\Gamma} g_{\mathrm{S}}^{2} C_{F}\left(\frac{-s_{12}-\imath 0}{\mu^{2}}\right)^{-\epsilon}\left[-\frac{2}{\epsilon^{2}}-\frac{3}{\epsilon}+\frac{8}{2 \epsilon-1}\right] \boldsymbol{S} \boldsymbol{p}_{\gamma \rightarrow q_{1} \bar{q}_{2}}^{(0)}
\end{aligned}
$$

for the QCD NLO corrections in the time-like region and using CDR scheme with $d=4-2 \epsilon$. It is interesting to notice that $\gamma \rightarrow q \bar{q}$ only involves rational functions (aside from the global prefactor) and is extremely simple. In the last part of this article, after presenting the full NLO corrections to triple-splitting functions started by photons, we will discuss the origin of these compact expressions, but we can anticipate that it is related with the kind of integrals involved in the computation. In the double collinear limit, it is difficult to appreciate this fact because there is only one kinematical scale involved $\left(s_{12}\right)$. In any case, since it is a three-particle process, computations can require triangle integrals at most. But only triangles with a LCG propagator can introduce hypergeometric functions which depend on $z_{1}$; otherwise the integral is completely independent of the momentum fraction. Moreover, the presence of momentum fractions inside non-rational functions is a consequence of having LCG propagators inside the loop integrals. Since this kind of functions is absent in $\boldsymbol{S p}_{\gamma \rightarrow q_{1} \bar{q}_{2}}^{(1)}$, then all LCG propagators inside the loop should cancel. As a partial summary of the situation, there is a connection between gauge constraints and having on-shell QCD particles (charged under a non-Abelian group) and just an off-shell parent photon (which is an Abelian field).

For the sake of completeness, we show the corresponding splitting functions for the double-collinear limit with photons. At LO we have

$$
\begin{aligned}
\left\langle\hat{P}_{q_{1} \gamma_{2}}^{(0)}\right\rangle & =g_{e}^{2} e_{q}^{2} \frac{1+z_{1}^{2}-\Delta\left(1-z_{1}\right)^{2}}{1-z_{1}}=g_{e}^{2} e_{q}^{2}\left\langle\mathcal{P}_{q_{1} \gamma_{2}}^{(0)}\right\rangle, \\
\left\langle\hat{P}_{\gamma \rightarrow q_{1} \bar{q}_{2}}^{(0)}\right\rangle & =g_{e}^{2} e_{q}^{2} \frac{C_{A}\left(1-2\left(1-z_{1}\right) z_{1}-\Delta\right)}{1-\Delta}=\frac{g_{e}^{2} e_{q}^{2} C_{A}}{1-\Delta}\left\langle\mathcal{P}_{\gamma \rightarrow q_{1} \bar{q}_{2}}^{(0)}\right\rangle,
\end{aligned}
$$

where $\Delta=\delta \epsilon$, with the parameter $\delta$ used to switch between $\mathrm{FDH} / \mathrm{HV}(\delta=0)$ and $\mathrm{CDR}$ 

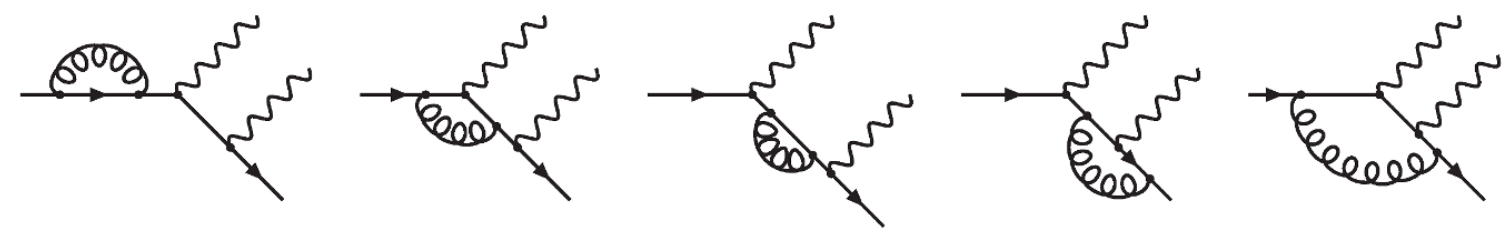

Figure 2. Representative Feynman diagrams contributing to the $q \rightarrow q \gamma \gamma$ splitting amplitude. This splitting amplitude is symmetric under the exchange of the two final state photons.

$(\delta=1)$ schemes. Choosing CDR scheme, the NLO corrections can be written as

$$
\begin{gathered}
\left\langle\hat{P}_{q_{1} \gamma_{2}}^{(1)}\right\rangle=c_{\Gamma} g_{\mathrm{S}}^{2} C_{F}\left(\frac{-s_{12}-\imath 0}{\mu^{2}}\right)^{-\epsilon}\left[\frac{2}{\epsilon^{2}}\left(1-{ }_{2} F_{1}\left(1,-\epsilon ; 1-\epsilon ; \frac{z_{1}-1}{z_{1}}\right)+\frac{\epsilon^{2}}{2(2 \epsilon-1)}\right)\left\langle\hat{P}_{q_{1} \gamma_{2}}^{(0)}\right\rangle\right. \\
\left.+g_{e}^{2} e_{q}^{2} \frac{z_{1}\left(1+z_{1}\right)}{\left(1-z_{1}\right)(1-2 \epsilon)}\right]+ \text { c.c. } \\
\left\langle\hat{P}_{\gamma \rightarrow q_{1} \bar{q}_{2}}^{(1)}\right\rangle=c_{\Gamma} g_{\mathrm{S}}^{2} C_{F}\left(\frac{-s_{12}-\imath 0}{\mu^{2}}\right)^{-\epsilon}\left[-\frac{2}{\epsilon^{2}}-\frac{3}{\epsilon}+\frac{8}{2 \epsilon-1}\right]\left\langle\hat{P}_{\gamma \rightarrow q_{1} \bar{q}_{2}}^{(0)}\right\rangle+\text { c.c. }
\end{gathered}
$$

where we adapted the original notation of [30] in order to be compatible with the one used in this article.

\section{$3.2 \quad q \rightarrow q \gamma \gamma$}

Let's consider the process $q \rightarrow q \gamma \gamma$. In terms of color structure, this is the simplest case because it is proportional to the identity matrix Id. The corresponding splitting amplitude at tree-level is given by

$$
\mathrm{Sp}_{q_{1} \gamma_{2} \gamma_{3}}^{(0)\left(a_{1} ; a\right)}=\frac{g_{e}^{2} e_{q}^{2} \mu^{2 \epsilon} \mathbf{I d}_{a_{1} a}}{s_{123} s_{13}} \bar{u}\left(p_{1}\right)\left(\notin\left(p_{3}\right) \not p_{3}+2 p_{1} \cdot \epsilon\left(p_{3}\right)\right) \notin\left(p_{2}\right) u(\widetilde{P})+(2 \leftrightarrow 3) .
$$

In order to reduce the output size and to show explicitly that the splitting function is dimensionless, we use the notation

$$
x_{i}=\frac{-s_{j k}-\imath 0}{-s_{123}-\imath 0}
$$

with $(i, j, k)$ a reordering of the indices set $\{1,2,3\}$ and the special case $x_{0} \equiv 1$. Moreover, we define

$$
\begin{aligned}
& \Delta_{i^{\prime}, j^{\prime}}^{i, j} \equiv x_{i} z_{j}+x_{i^{\prime}}\left(z_{j^{\prime}}-1\right), \\
& \bar{\Delta}_{i^{\prime}, j^{\prime}}^{i, j} \equiv x_{i} z_{j}-x_{i^{\prime}}\left(z_{j^{\prime}}-1\right),
\end{aligned}
$$

where the indices represent outgoing particles, and $z_{0} \equiv 1$. Using this notation, it is possible to write the unpolarized splitting function at $\mathrm{LO}$ as

$$
\begin{aligned}
\left\langle\hat{P}_{q_{1} \gamma_{2} \gamma_{3}}^{(0)}\right\rangle= & \frac{e_{q}^{4} g_{e}^{4}}{x_{2}}\left(\frac{\left\langle\mathcal{P}_{q_{1} \gamma_{2}}^{(0)}\right\rangle}{z_{2}}\left(1+\frac{\left(1+x_{2}\right) z_{1}}{x_{3}}\right)+(\Delta-1)\left(\Delta\left(x_{1}-\frac{z_{1}\left(x_{1}+1\right)}{1-x_{1}}+\frac{z_{1}}{2 x_{3}}\right)\right.\right. \\
& \left.\left.+x_{3}+z_{1}-z_{2}+2\right)\right)+(2 \leftrightarrow 3) .
\end{aligned}
$$


Going to the NLO correction, we use the decomposition suggested in eq. (2.14) and eq. (2.15). The factor predicted by Catani's formula is

$$
I_{q_{1} \gamma_{2} \gamma_{3}}^{(1)}\left(p_{1}, p_{2}, p_{3} ; \widetilde{P}\right)=2 c_{\Gamma} g_{\mathrm{S}}^{2} C_{F}\left(\frac{-s_{123}-\imath 0}{\mu^{2}}\right)^{-\epsilon} \frac{1-z_{1}^{-\epsilon}}{\epsilon^{2}}
$$

and we found agreement with the divergent contribution in our results.

The rational coefficient is given by

$$
\begin{aligned}
C^{(0)}= & \frac{\left\langle\mathcal{P}_{q_{1} \gamma_{2}}^{(0)}\right\rangle\left(z_{1}-1\right)\left(x_{1}\left(x_{2}-1\right)+\left(x_{3}-1\right) z_{2}\right)}{x_{1}^{2} x_{2}\left(x_{3}-1\right) z_{2}}+\frac{\left(x_{1}-1\right)\left(\Delta_{0,1}^{1,0}-3\right)}{x_{1} x_{2} z_{2}}+\frac{3 x_{1}^{2} \bar{\Delta}_{0,2}^{3,0}-z_{2}}{x_{1}^{2}\left(1-x_{2}\right) x_{2}} \\
& +\frac{\left(\Delta_{0,1}^{1,0}\right)^{2}+2 x_{1} z_{2}+2\left(1-x_{1}\right)^{2}}{x_{1}^{2} x_{2}}+\frac{\Delta_{0,1}^{1,2}-x_{1} z_{3}}{x_{1}^{2}\left(1-x_{2}\right)}+\frac{z_{1}\left(x_{1}^{2}+2\left(x_{1}+1\right) x_{3}\right)}{x_{1}^{2}\left(x_{3}-1\right) x_{3} z_{2}} \\
& +\frac{1}{x_{1}^{2} z_{2}}\left(\frac{x_{1}^{3}}{1-x_{2}}+\frac{2 z_{1}}{x_{2}}\right)-\frac{3\left(1-x_{2}\right) z_{1}}{x_{1} x_{2} x_{3} z_{2}}+\frac{4\left(z_{1}-1\right)}{x_{1} z_{2}}+(2 \leftrightarrow 3),
\end{aligned}
$$

with the global prefactor

$$
c^{q \gamma \gamma}=e_{q}^{4} g_{e}^{4} g_{\mathrm{S}}^{2} C_{F} .
$$

At transcendental weight 1 , the basis is composed by

$$
\begin{aligned}
& F_{1}^{(1)}=\log \left(x_{1}\right), \\
& F_{2}^{(1)}=\log \left(x_{3}\right) .
\end{aligned}
$$

It is important to notice that these logarithms depend on ratios of kinematical variables, which ensures that this contribution can be integrated if we need to compute $\mathrm{N}^{3} \mathrm{LO}$ corrections. Due to symmetry considerations, we can represent the results as

$$
\left.\left\langle\hat{P}_{q_{1} \gamma_{2} \gamma_{3}}^{(1) \text { fin. }}\right\rangle\right|_{w=1}=c^{q \gamma \gamma}\left[\sum_{i=1}^{2} C_{i}^{(1)} F_{i}^{(1)}+(2 \leftrightarrow 3)\right],
$$

where

$$
\begin{aligned}
C_{1}^{(1)}= & \frac{2 \Delta_{2,3}^{0,1}\left(\left(z_{3}-1\right) \Delta_{2,3}^{0,1}+x_{3}\left(z_{1}+z_{3}\left(2 z_{2}+z_{3}-2\right)+1\right)\right)}{\left(1-x_{1}\right) x_{3}^{2} z_{2} z_{3}}+\frac{4 x_{2} \bar{\Delta}_{0,3}^{3,0}}{x_{3}\left(1-x_{1}\right)} \\
& +\frac{2 z_{2} z_{3}\left(x_{1}+z_{1}\right)+\left(z_{1}+1\right) z_{1}}{\left(x_{1}-1\right) z_{2} z_{3}}, \\
C_{2}^{(1)}= & \frac{\Delta_{0,1}^{1,0}}{1-x_{3}}\left(\frac{2\left(\Delta_{0,1}^{1,0}-z_{1}+1\right)}{x_{1} x_{2}\left(1-z_{1}\right) z_{2}}+\frac{\Delta_{0,1}^{1,0}+2 z_{1}}{x_{1} x_{3} z_{3}}+\frac{1}{x_{1} z_{2}}-\frac{x_{2}\left(1-z_{1}\right)-\left(1-x_{3}\right)\left(5 z_{1}-2\right)}{x_{2}\left(1-x_{3}\right)\left(1-z_{1}\right) z_{2}}\right. \\
& \left.-\frac{\Delta_{0,1}^{1,0}}{\left(1-z_{1}\right) z_{2}}\left(\frac{z_{1}\left(3 x_{1}+4 x_{2}\right)+x_{1}-z_{1}+1}{x_{1} x_{2}\left(1-x_{3}\right)}+\frac{2}{x_{2}^{2}}\right)-4\right) \\
& +\frac{1}{1-x_{3}}\left(2 \Delta_{0,2}^{2,0}-\frac{3 x_{1}^{2} \Delta_{0,3}^{1,0}+x_{1}\left(z_{2}-z_{3}\right)+z_{2}+2 z_{3}}{x_{1}^{2}\left(1-x_{3}\right)}+\frac{1}{z_{2}}\left(\frac{3\left(1-z_{1}\right) z_{1}}{x_{1}}-\frac{2 \Delta_{0,1}^{1,1}}{x_{1}\left(1-x_{3}\right)}\right)\right.
\end{aligned}
$$




$$
\begin{aligned}
& +\frac{x_{1}\left(x_{1}^{2}\left(z_{1}-z_{2}+2\right)-x_{1}\left(z_{3}+1\right)+4 z_{1}+z_{2}-2\right)-2 z_{1}-z_{2}+2}{x_{1}^{2} x_{2}}+\frac{x_{1}}{\left(1-x_{3}\right) z_{3}} \\
& \left.+\frac{x_{1} z_{1}^{2}\left(2 x_{1} z_{1}+x_{2}\left(z_{1}-2\right)\right)}{x_{2}^{2}\left(z_{1}-1\right) z_{3}}-\frac{\left(x_{1}+3\right) z_{1}^{2}-4 z_{1}+1}{x_{1} z_{3}}-2\right) \\
& +\frac{1-x_{1}}{1-x_{3}}\left(\frac{2\left(x_{1}+z_{1}\right)-z_{2}-\bar{\Delta}_{0,1}^{1,3}}{x_{1} x_{3}}-\frac{2\left(\Delta_{0,1}^{1,1}-\bar{\Delta}_{0,2}^{1,2}+2 x_{1}\right)}{x_{2}^{2}}\right)
\end{aligned}
$$

The splitting is fully symmetric under the exchange of particles 2 and 3 , then let's exploit this symmetry to minimize the basis. At weight 2 :

$$
\left.\left\langle\hat{P}_{q_{1} \gamma_{2} \gamma_{3}}^{(1) \mathrm{fin}}\right\rangle\right|_{w=2}=c^{q \gamma \gamma}\left[\sum_{i=1}^{7} C_{i}^{(2)} F_{i}^{(2)}+(2 \leftrightarrow 3)\right],
$$

where the corresponding basis is

$$
\begin{aligned}
F_{1}^{(2)}= & \log ^{2}\left(z_{1}\right), \\
F_{2}^{(2)}= & \frac{\pi^{2}}{6}-\operatorname{Li}_{2}\left(1-x_{1}\right) \\
F_{3}^{(2)}= & \log \left(x_{1}\right) \log \left(z_{1}\right), \\
F_{4}^{(2)}= & \operatorname{Li}_{2}\left(1-x_{2}\right)+\log \left(x_{1}\right) \log \left(x_{2}\right), \\
F_{5}^{(2)}= & \mathcal{R}\left(x_{1}, x_{2}\right)+2 \operatorname{Li}_{2}\left(1-x_{2}\right)+2 \log \left(x_{1}\right) \log \left(x_{2}\right), \\
F_{6}^{(2)}= & \operatorname{Li}_{2}\left(\frac{\Delta_{0,2}^{2,0}}{z_{2}-1}\right)-\operatorname{Li}_{2}\left(1-x_{2}\right)-\operatorname{Li}_{2}\left(-\frac{z_{3}}{z_{1}}\right)-\operatorname{Li}_{2}\left(\frac{z_{2}}{z_{2}-1}\right) \\
& -\log \left(x_{2}\right) \log \left(\frac{1-z_{2}}{z_{1}}\right), \\
F_{7}^{(2)}= & \operatorname{Li}_{2}\left(z_{2}\right)+\log \left(\frac{1-z_{2}}{z_{1}}\right) \log \left(\frac{x_{1}\left(1-z_{2}\right)}{x_{2}}\right)+\log \left(x_{1}\right) \log \left(z_{1}\right),
\end{aligned}
$$

with $\Delta_{0,2}^{2,0}=x_{2}+z_{2}-1$. The first three components of the basis are symmetric under the exchange of particles 2 and 3. On the other hand, in the previous list we introduced the function

$$
\mathcal{R}\left(x_{1}, x_{2}\right)=\frac{\pi^{2}}{6}-\log \left(x_{1}\right) \log \left(x_{2}\right)-\operatorname{Li}_{2}\left(1-x_{1}\right)-\operatorname{Li}_{2}\left(1-x_{2}\right),
$$

which is going to appear in all the remaining splitting functions, and whose origin will be explained in the last part of this article. Also, we can appreciate that $\mathcal{R}\left(x_{i}, x_{j}\right)=0$ when $x_{k} \rightarrow 0(k \neq i, j)$. Following with the presentation of the results, the coefficients associated with the previous basis are given by

$$
\begin{aligned}
& C_{1}^{(2)}=\frac{2 z_{1}\left(z_{2} z_{3}+\left(1-z_{2}\right)^{2}\right)}{x_{2} x_{3} z_{2} z_{3}} \\
& C_{2}^{(2)}=\frac{4\left(1-z_{2}\right)\left(\bar{\Delta}_{1,2}^{0,3}\right)^{2}}{x_{2}^{3} z_{2} z_{3}}-\frac{16 x_{1}\left(1-z_{2}\right)^{2}}{x_{2}^{3} z_{2}}-\frac{2\left(1-x_{1}\right)^{2}\left(3 x_{1} z_{2} z_{3}+z_{1}\left(z_{1}^{2}+1\right)\right)}{x_{1} x_{2} x_{3} z_{2} z_{3}}
\end{aligned}
$$




$$
\begin{aligned}
& +\frac{4 z_{1}\left(2\left(1-z_{2}\right) z_{3}-\bar{\Delta}_{1,2}^{0,3}\right)}{x_{2}^{2} z_{2} z_{3}}+\frac{4\left(z_{2}\left(x_{1}-2 z_{1}-z_{3}\right)+\left(1-z_{3}\right)^{2}\right)}{x_{3} z_{2}}+\frac{4\left(1-z_{3}\right)^{2}}{x_{2} z_{2}}, \\
C_{3}^{(2)}= & \frac{4\left(z_{1}\left(1-z_{2}\right)+\left(1-z_{3}\right)^{3}\right)}{x_{3} z_{2} z_{3}}-\frac{4 x_{2}}{x_{3}},
\end{aligned}
$$

for the explicitly symmetric contribution, and

$$
\begin{aligned}
C_{4}^{(2)}= & -\frac{2\left(x_{3} z_{1}-\Delta_{0,2}^{2,0}-x_{1} z_{3}\right)\left(\left(z_{1}^{2}+1\right) \Delta_{3,2}^{2,2}-x_{1} z_{2} z_{3}\left(2 x_{2}+x_{3}\right)\right)}{x_{1} x_{2} x_{3} z_{2} z_{3} \Delta_{0,2}^{2,0}} \\
C_{5}^{(2)}= & -\frac{2\left\langle\mathcal{P}_{q_{1} \gamma_{2}}^{(0)}\right\rangle\left(x_{2} z_{2} \bar{\Delta}_{0,1}^{1,0}+x_{3}\left(z_{1}-1\right)\left(x_{1}\left(z_{2}-1\right)+z_{1}-z_{2}+1\right)\right)}{x_{1} x_{2} x_{3} z_{2} z_{3}}+\frac{2 \Delta_{0,1}^{1,0}\left(\Delta_{0,1}^{1,0}-x_{3} z_{1}\right)}{x_{3}^{3}\left(z_{1}-1\right) z_{3}} \\
& -\frac{2 x_{1}^{2} z_{1}^{3}-2 x_{1} x_{3} z_{1}^{2}+2 x_{3}^{2}\left(z_{1}+1\right) z_{1}}{x_{3}^{3}\left(1-z_{1}\right) z_{2}}+\frac{4\left(1-x_{1}\right)\left(2 x_{2}+x_{3}\right)}{x_{2} x_{3}} \\
& -\frac{2\left(1-x_{1}\right)\left(x_{1}\left(2 z_{1}+z_{2}+1\right)-z_{2}-1\right)}{x_{3}^{3}}+\frac{4 z_{1}}{x_{3}^{2}}+\frac{2}{x_{3} z_{3}}-4 \\
C_{6}^{(2)}= & -\frac{2\left\langle\mathcal{P}_{q_{1} \gamma_{2}}^{(0)}\right\rangle\left(1-z_{1}\right)\left(\left(z_{1}-1\right) \Delta_{0,3}^{3,0}+x_{1}\left(z_{1}-z_{2}\right)-z_{1} z_{3}\right)}{x_{1} x_{2} x_{3} z_{2} z_{3}}, \\
C_{7}^{(2)}= & \frac{2\left\langle\mathcal{P}_{q_{1} \gamma_{2}}^{(0)}\right\rangle}{x_{2} x_{3} \Delta_{0,2}^{2,0}}\left(\frac{x_{2} \Delta_{0,1}^{1,0}+x_{2} x_{3}\left(1-z_{1}\right)+2 x_{3} z_{1}^{2}}{z_{3}}+\frac{x_{1} x_{2} z_{1}+x_{2} x_{3}\left(z_{1}-1\right)+2 x_{3}}{z_{2}}\right) \\
& -\frac{2\left(x_{1}\left(z_{2}-1\right)+2 z_{1}^{2}+z_{1}\left(3-2 z_{2}\right)+2 z_{2}^{2}-7 z_{2}+7\right)}{x_{2} \Delta_{0,2}^{2,0}}+\frac{4 \Delta_{1,3}^{0,2}}{x_{3} \Delta_{0,2}^{2,0}} \\
& +\frac{2\left(z_{1}-2 z_{2}+3\right)}{\Delta_{0,2}^{2,0}}, \quad
\end{aligned}
$$

for the others.

\section{$3.3 \quad q \rightarrow q g \gamma$}

The natural following step is to replace one photon by a gluon. This will produce more complicated expressions, but it is possible to relate them with the $q \rightarrow q \gamma \gamma$ splitting. In order to do that, let's take a look at figure 3. At LO, we have exactly the same kinematic structure, which implies that

$$
\begin{aligned}
\operatorname{Sp}_{q_{1} g_{2} \gamma_{3}}^{(0)\left(a_{1}, \alpha_{2} ; a\right)} & =\frac{e_{q} g_{e} \mu^{2 \epsilon} \boldsymbol{T}_{a_{1} a}^{\alpha_{2}}}{s_{123}} \bar{u}\left(p_{1}\right)\left[\left(\notin\left(p_{3}\right) p_{3}+2 p_{1} \cdot \epsilon\left(p_{3}\right)\right) \frac{\notin\left(p_{2}\right)}{s_{13}}+(2 \leftrightarrow 3)\right] u(\widetilde{P}) \\
& =\boldsymbol{T}_{a_{1} a}^{\alpha_{2} a} \frac{g_{\mathrm{S}}}{e_{q} g_{e}} \operatorname{Sp}_{q_{1} \gamma_{2} \gamma_{3}}^{(0)\left(a_{1} ; a\right)}
\end{aligned}
$$

and the corresponding unpolarized LO splitting function is simply

$$
\left\langle\hat{P}_{q_{1} g_{2} \gamma_{3}}^{(0)}\right\rangle=C_{F} \frac{g_{\mathrm{S}}^{2}}{e_{q}^{2} g_{e}^{2}}\left\langle\hat{P}_{q_{1} \gamma_{2} \gamma_{3}}^{(0)}\right\rangle,
$$

in terms of the one associated to $q \rightarrow q \gamma \gamma$. 

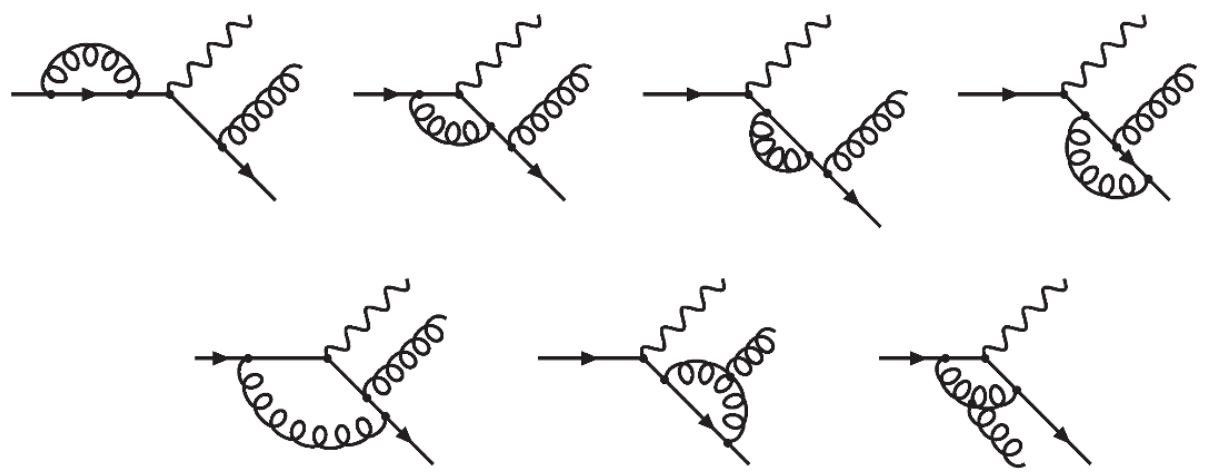

Figure 3. Representative Feynman diagrams contributing to the $q \rightarrow q g \gamma$ splitting amplitude. The first 5 diagrams are associated with the Abelian contribution already presented in $q \rightarrow q \gamma \gamma$. The last 2 diagrams lead to non-Abelian (NA) contributions.

To obtain the NLO corrections, we use the decomposition suggested in eq. (2.14) and eq. (2.15). The factor predicted by Catani's formula is

$$
\begin{aligned}
I_{q_{1} g_{2} \gamma_{3}}^{(1)}\left(p_{1}, p_{2}, p_{3} ; \widetilde{P}\right) & =\frac{c_{\Gamma} g_{\mathrm{S}}^{2}}{\epsilon^{2}}\left(\frac{-s_{123}-\imath 0}{\mu^{2}}\right)^{-\epsilon}\left[2 C_{F}-C_{A}\left(x_{3}^{-\epsilon}+z_{2}^{-\epsilon}\right)-D_{A} z_{1}^{-\epsilon}\right](3.4 \\
& =\frac{D_{A}}{2 C_{F}} I_{q_{1} \gamma_{2} \gamma_{3}}^{(1)}\left(p_{1}, p_{2}, p_{3} ; \widetilde{P}\right)+C_{A} I_{q_{1} g_{2} \gamma_{3}}^{\left(1, C_{A}\right)}\left(p_{1}, p_{2}, p_{3} ; \widetilde{P}\right)
\end{aligned}
$$

where

$$
I_{q_{1} g_{2} \gamma_{3}}^{\left(1, C_{A}\right)}\left(p_{1}, p_{2}, p_{3} ; \widetilde{P}\right)=\frac{c_{\Gamma} g_{\mathrm{S}}^{2}}{\epsilon^{2}}\left(\frac{-s_{123}-\imath 0}{\mu^{2}}\right)^{-\epsilon}\left(1-x_{3}^{-\epsilon}-z_{2}^{-\epsilon}\right),
$$

with $D_{A}=2 C_{F}-C_{A}$ and we found agreement with the divergent contribution in our results.

Before writing explicit results, let's take a look to the corresponding NLO Feynman diagrams contributing to the splitting amplitude. If we just consider the topology, we can appreciate that many diagrams are already present in figure 2. Since a photon has been replaced by a gluon, there is an extra color matrix. But in these diagrams, the kinematic structure is unchanged and only some color factors need to be modified. At amplitude level, in $q \rightarrow q \gamma \gamma$ there was a global color factor $\left(\boldsymbol{T}^{\beta} \boldsymbol{T}^{\beta}\right)_{a_{1} a}=C_{F} \mathbf{I d}_{a_{1} a}$ related with a gluon of color $\beta$ inside the loop. However, in $q \rightarrow q g \gamma$ we must insert the color matrix $\boldsymbol{T}^{\alpha}$ associated with the external gluon, which leads to two possibilities:

- $\left(\boldsymbol{T}^{\beta} \boldsymbol{T}^{\beta} \boldsymbol{T}^{\alpha}\right)_{a_{1} a}=\frac{D_{A}+C_{A}}{2} \boldsymbol{T}_{a_{1} a}^{\alpha}$ if the external gluon is attached to a fermion line inside the loop, or;

- $\left(\boldsymbol{T}^{\beta} \boldsymbol{T}^{\alpha} \boldsymbol{T}^{\beta}\right)_{a_{1} a}=\frac{D_{A}}{2} \boldsymbol{T}_{a_{1} a}^{\alpha}$ when the external gluon interacts with an external fermion (i.e. a fermionic line which is not inside a loop).

It is important to notice that both configurations include a contribution proportional to $C_{F}$. On the other hand, there are some diagrams which include a triple-gluon vertex in figure 3 whose topology is different from the ones associated with the $q \rightarrow q \gamma \gamma$ process. 
In summary, we conclude that it is possible to express the $q \rightarrow q g \gamma$ splitting function in terms of $q \rightarrow q \gamma \gamma$. To make this relation explicit at NLO, we write

$$
\left\langle\hat{P}_{q_{1} g_{2} \gamma_{3}}^{(1)}\right\rangle=D_{A}\left\langle\hat{P}_{q_{1} g_{2} \gamma_{3}}^{\left(1, D_{A}\right)}\right\rangle+C_{A}\left\langle\hat{P}_{q_{1} g_{2} \gamma_{3}}^{\left(1, C_{A}\right)}\right\rangle+\text { c.c. },
$$

with

$$
\begin{aligned}
\left\langle\hat{P}_{q_{1} g_{2} \gamma_{3}}^{\left(1, D_{A}\right)}\right\rangle & =\frac{1}{2 C_{F}}\left[\operatorname{Re}\left(I_{q_{1} \gamma_{2} \gamma_{3}}^{(1)}\left(p_{1}, p_{2}, p_{3} ; \widetilde{P}\right)\right)\left\langle\hat{P}_{q_{1} g_{2} \gamma_{3}}^{(0)}\right\rangle+\left\langle\hat{P}_{q_{1} \gamma_{2} \gamma_{3}}^{(1)) \text { fin. }}\right\rangle\right], \\
\left\langle\hat{P}_{q_{1} g_{2} \gamma_{3}}^{\left(1, C_{A}\right)}\right\rangle & =\operatorname{Re}\left(I_{q_{1} g_{2} \gamma_{3}}^{\left(1, C_{A}\right)}\left(p_{1}, p_{2}, p_{3} ; \widetilde{P}\right)\right)\left\langle\hat{P}_{q_{1} g_{2} \gamma_{3}}^{(0)}\right\rangle+\left\langle\hat{P}_{q_{1} g_{2} \gamma_{3}}^{\left(1, C_{A}\right) \text { fin. }}\right\rangle,
\end{aligned}
$$

where the global prefactor is given by

$$
c^{q g \gamma}=e_{q}^{2} g_{e}^{2} g_{\mathrm{S}}^{4} C_{F} .
$$

Centering in the finite NLO contribution, we can write the corrections proportional to $C_{A}$ using eq. (2.15) as

$$
\left\langle\hat{P}_{q_{1} g_{2} \gamma_{3}}^{\left(1, C_{A}\right) \text { fin. }}\right\rangle=c^{q g \gamma}\left[C^{\left(0, C_{A}\right)}+\sum_{i=1}^{2} \sum_{j} C_{j}^{\left(i, C_{A}\right)} F_{j}^{\left(i, C_{A}\right)}\left(\left\{x_{l}, z_{k}\right\}\right)+\mathcal{O}(\epsilon)\right] .
$$

After these considerations, let's show the explicit results. In first place, the rational coefficient is given by

$$
\begin{aligned}
C^{\left(0, C_{A}\right)}= & \frac{x_{1}\left(z_{2}\left(\Delta_{0,3}^{3,0}+4\left(1-x_{2} z_{3}\right)\right)+3 x_{3} z_{3}\right)+x_{2} z_{3}\left(3 z_{2}-4 x_{3}\right)}{2 x_{1}\left(x_{2}-1\right) x_{2} z_{3}}+\frac{\left(2-x_{1}\right) x_{1}^{2}+x_{2}\left(x_{3}-1\right) z_{2}}{2 x_{1}\left(x_{2}-1\right) x_{2} x_{3} z_{2}^{-1} z_{3}} \\
& -\frac{\left(1-x_{3}\right)\left(4\left(1-x_{2}\right)-x_{1}\left(3 x_{1}+x_{3}\right)\right)-x_{1}\left(x_{1}+1\right) x_{3}}{2\left(x_{2}-1\right) x_{2}\left(x_{3}-1\right) x_{3} z_{2}}-(2 \leftrightarrow 3),
\end{aligned}
$$

which is totally antisymmetric when interchanging particles 2 and 3 . Since the rational contribution for the $q \rightarrow q \gamma \gamma$ kernel is totally symmetric, it is clear that $C^{\left(0, C_{A}\right)}$ is related with the NA diagrams associated with the non-Abelian triple-vertex.

At weight 1 , the basis is composed by

$$
F^{\left(1, C_{A}\right)}=\log \left(x_{3}\right),
$$

which only depend on ratios of kinematical variables. Due to symmetry considerations, we can rewrite this contribution as

$$
\left.\left\langle\hat{P}_{q_{1} g_{2} \gamma_{3}}^{\left(1, C_{A}\right) \text { fin. }}\right\rangle\right|_{w=1}=c^{q g \gamma}\left[\left(C_{\mathrm{sym}}^{\left(1, C_{A}\right)} F^{\left(1, C_{A}\right)}+(2 \leftrightarrow 3)\right)+\left(C_{\mathrm{asym}}^{\left(1, C_{A}\right)} F^{\left(1, C_{A}\right)}-(2 \leftrightarrow 3)\right)\right],
$$

where

$$
\begin{aligned}
C_{\mathrm{sym}}^{\left(1, C_{A}\right)}= & \frac{3\left(x_{1}\left(1-\Delta_{0,1}^{1,0}-\bar{\Delta}_{1,2}^{1,1}-z_{2}\right)+z_{1}\right)}{2 x_{1} x_{2}\left(1-x_{3}\right)}+\frac{3\left(z_{3} \Delta_{3,1}^{1,1}\left(\Delta_{0,1}^{1,0}-z_{1}^{2}+1\right)+x_{1}^{2} z_{1}^{3} z_{2}\right)}{2 x_{1} x_{2}\left(x_{3}-1\right)\left(z_{1}-1\right) z_{2} z_{3}} \\
& +\frac{3 z_{1}\left(\Delta_{0,1}^{1,0}-x_{3} \Delta_{0,1}^{1,1}\right)}{2 x_{1}\left(1-x_{3}\right) x_{3} z_{3}}+\frac{3\left(2 \Delta_{0,2}^{2,0}-z_{1}-1\right)}{2\left(1-x_{3}\right)}+\frac{3\left(1-x_{1}\right) z_{1}}{2 x_{1}\left(1-x_{3}\right) x_{3}}
\end{aligned}
$$




$$
\begin{aligned}
C_{\mathrm{asym}}^{\left(1, C_{A}\right)}= & \frac{\left\langle\mathcal{P}_{q_{1} \gamma_{2}}^{(0)}\right\rangle\left(z_{1}-1\right)}{x_{1}^{2} x_{3} z_{3}}\left(\frac{x_{2}}{1-x_{3}}-x_{2}-1\right)+\frac{\left(2 x_{1}-3\right)\left(\Delta_{1,3}^{0,2}+2 \Delta_{0,1}^{1,0}\right)}{2 x_{1}^{2} x_{2}\left(1-x_{3}\right)} \\
& -\frac{x_{1}\left(3 x_{1} \Delta_{0,3}^{1,0}+z_{2}-z_{3}\right)+z_{2}+2 z_{3}}{2 x_{1}^{2}\left(1-x_{3}\right)^{2}}+\frac{\left(1-z_{1}\right)\left(2\left(\bar{\Delta}_{3,1}^{1,0}-z_{1}+1\right)-5 x_{1}\right)}{2 x_{1}^{2}\left(1-x_{3}\right) z_{2}} \\
& +\frac{\left(x_{1}-1\right)\left(x_{1}^{2}\left(z_{3}-2\right)-x_{1}\left(2 z_{1}+z_{2}+1\right)-2 z_{1}+2 z_{2}+2\right)}{2 x_{1}^{2}\left(1-x_{3}\right) x_{3}} \\
& +\frac{x_{1}\left(z_{1}-1\right)\left(2 x_{3} z_{1}+x_{3}-3\right)-4 z_{1}\left(x_{2} x_{3}+x_{3}-1\right)}{2 x_{1}^{2}\left(x_{3}-1\right) x_{3} z_{3}} \\
& -\frac{x_{2} \Delta_{0,1}^{1,1} \bar{\Delta}_{0,1}^{1,0}+\left(2 x_{1}-3\right)\left(1-x_{3}\right)\left(1-z_{1}\right) \Delta_{0,1}^{1,0}}{2 x_{1}^{2} x_{2}\left(1-x_{3}\right)^{2} z_{2}} \\
& +\frac{x_{1}+\left(x_{3}-1\right)\left(x_{3}\left(z_{1}-1\right) z_{3}-z_{1}\right)}{2\left(x_{3}-1\right)^{2} x_{3} z_{3}}
\end{aligned}
$$

Finally, if we go to transcendental weight 2 , it is possible to perform the expansion

$$
\left.\left\langle\hat{P}_{q_{1} g_{2} \gamma_{3}}^{\left(1, C_{A}\right) \text { fin. }}\right\rangle\right|_{w=2}=c^{q g \gamma} \sum_{i=1}^{7} C_{i}^{\left(2, C_{A}\right)} F_{i}^{\left(2, C_{A}\right)},
$$

using the set of functions

$$
\begin{aligned}
F_{1}^{\left(2, C_{A}\right)}= & \operatorname{Li}_{2}\left(z_{3}\right)+\log \left(1-z_{3}\right) \log \left(\frac{x_{2}\left(1-z_{3}\right)}{z_{2}}\right)+\log \left(x_{3}\right) \log \left(\frac{z_{2}}{1-z_{3}}\right), \\
F_{2}^{\left(2, C_{A}\right)}= & \frac{\pi^{2}}{6}-\operatorname{Li}_{2}\left(1-x_{2}\right) \\
F_{3}^{\left(2, C_{A}\right)}= & \mathrm{Li}_{2}\left(1-x_{3}\right)-\mathrm{Li}_{2}\left(z_{3}\right)+\log \left(\frac{1-z_{3}}{x_{3}}\right) \log \left(\frac{z_{2}}{x_{2}\left(1-z_{3}\right)}\right), \\
F_{4}^{\left(2, C_{A}\right)}= & \frac{\pi^{2}}{6}-\mathrm{Li}_{2}\left(1-x_{2}\right)-\operatorname{Li}_{2}\left(1-z_{2}\right)+\log \left(x_{2}\right) \log \left(z_{2}\right) \\
F_{5}^{\left(2, C_{A}\right)}= & 2\left[\operatorname{Li}_{2}\left(1-x_{3}\right)+\operatorname{Li}_{2}\left(-\frac{z_{1}}{z_{2}}\right)-\operatorname{Li}_{2}\left(-\frac{\Delta_{0,3}^{3,0}}{1-z_{3}}\right)\right]-\operatorname{Li}_{2}\left(z_{3}\right) \\
& +\log \left(x_{2}\right) \log \left(1-z_{3}\right)+\log \left(\frac{x_{3}}{z_{2}}\right) \log \left(\frac{1-z_{3}}{z_{2}}\right) \\
F_{6}^{\left(2, C_{A}\right)}= & \frac{\pi^{2}}{6}-\operatorname{Li}_{2}\left(1-x_{2}\right)-\operatorname{Li}_{2}\left(1-z_{2}\right) \\
F_{7}^{\left(2, C_{A}\right)}= & \mathcal{R}\left(x_{2}, x_{3}\right)
\end{aligned}
$$

where the associated coefficients are given by

$$
\begin{aligned}
C_{1}^{\left(2, C_{A}\right)}= & \frac{\left\langle\mathcal{P}_{q_{1} \gamma_{2}}^{(0)}\right\rangle}{1-x_{1}}\left(\frac{\left(x_{1}-3\right) z_{1}}{x_{3} z_{2}}-\frac{1-x_{1}+2 z_{1}}{x_{2} z_{2}}-\frac{\left(1-x_{1}\right) z_{1}+2}{x_{2} z_{3}}+\frac{x_{1}-3}{x_{3} z_{3}}\right) \\
& +\frac{4}{x_{2}}+\frac{2\left(4-z_{2}-2 z_{3}\right)}{x_{3}}, \\
C_{2}^{\left(2, C_{A}\right)}= & \frac{\left\langle\mathcal{P}_{q_{1} \gamma_{2}}^{(0)}\right\rangle}{1-x_{1}}\left(\frac{3 x_{1}+z_{1}-3}{x_{2} z_{2}}+\frac{\left(3 x_{1}-4\right) z_{1}+2}{x_{2} z_{3}}+\frac{\left(3 x_{1}-2\right) z_{1}}{x_{3} z_{2}}+\frac{3 x_{1}-z_{1}-1}{x_{3} z_{3}}\right)
\end{aligned}
$$




$$
\begin{aligned}
& +\frac{2\left(2\left(1-x_{1}+z_{1}\right)+z_{2}\right)}{x_{3}}-\frac{4\left(x_{1}-z_{1}+z_{2}-2\right)}{x_{2}}-8, \\
& C_{3}^{\left(2, C_{A}\right)}=\frac{\left\langle\mathcal{P}_{q_{1} \gamma_{2}}^{(0)}\right\rangle}{\left(1-x_{1}\right) \Delta_{0,3}^{3,0}}\left(\frac{z_{1} \Delta_{0,1}^{1,0}}{x_{2} z_{2}}+\frac{\bar{\Delta}_{1,1}^{1,0}}{x_{2} z_{3}}+\frac{\bar{\Delta}_{0,1}^{1,0}}{x_{3} z_{3}}-\frac{\left(1-x_{1}^{2}\right)\left(1-z_{1}\right)}{x_{2} x_{3}}+\frac{x_{1} z_{1}^{2}}{x_{3} z_{2}}\right) \\
& -\frac{2\left(x_{1}\left(z_{2}+1\right)-z_{3}\right)}{x_{2} \Delta_{0,3}^{3,0}}-\frac{x_{1}\left(1-z_{3}\right)+z_{2}}{x_{3} \Delta_{0,3}^{3,0}}-\frac{1-z_{1}}{\Delta_{0,3}^{3,0}}, \\
& C_{4}^{\left(2, C_{A}\right)}=\frac{\left\langle\mathcal{P}_{q_{1} \gamma_{2}}^{(0)}\right\rangle}{z_{2} z_{3} \Delta_{0,2}^{2,0}}\left(\left(1-z_{1}\right)\left(z_{3}-z_{2}\right)-\frac{z_{2} \Delta_{0,1}^{1,0}+x_{1} z_{1} z_{3}}{x_{3}}\right)+\frac{x_{1}\left(z_{2}-1\right)+z_{3}}{x_{2} \Delta_{0,2}^{2,0}} \\
& -\frac{2\left(\left(1-x_{1}\right)\left(2-x_{1}-z_{3}\right)-z_{1}+\left(1-z_{3}\right)^{2}\right)}{x_{3} \Delta_{0,2}^{2,0}}+\frac{2\left(2 x_{2}+3 z_{2}\right)-3\left(1-z_{1}\right)}{\Delta_{0,2}^{2,0}}, \\
& C_{5}^{\left(2, C_{A}\right)}=\frac{z_{1}\left(z_{1}^{2}+1\right)}{2 x_{2} x_{3} z_{2} z_{3}}+(2 \leftrightarrow 3), \\
& C_{6}^{\left(2, C_{A}\right)}=-\frac{2\left\langle\mathcal{P}_{q_{1} \gamma_{2}}^{(0)}\right\rangle}{x_{2} \Delta_{0,2}^{2,0}}\left(\frac{z_{1}^{2}}{z_{3}}+\frac{1}{z_{2}}\right)+\frac{2\left(\left(1-x_{1}\right)^{2}+\left(1-z_{3}\right)^{2}\right)}{x_{3} \Delta_{0,2}^{2,0}} \\
& -\frac{4 \bar{\Delta}_{0,3}^{2,0}}{\Delta_{0,2}^{2,0}}+\frac{2\left(z_{1}\left(4-3 z_{2}\right)-z_{2}+z_{3}^{2}+2\right)}{x_{2} \Delta_{0,2}^{2,0}}, \\
& C_{7}^{\left(2, C_{A}\right)}=\frac{\left\langle\mathcal{P}_{q_{1} \gamma_{2}}^{(0)}\right\rangle}{x_{1}^{3} x_{3}}\left(\frac{x_{1}^{3}\left(x_{2} z_{1}+x_{3}\right)+x_{3}^{3}\left(1-z_{1}\right)}{x_{2} z_{2}}+\frac{\Delta_{0,1}^{1,0}+x_{1}^{3}\left(z_{1}-x_{1}\right)+2 x_{1}}{z_{3}\left(1-x_{1}\right)}\right) \\
& -\frac{\left\langle\mathcal{P}_{q_{1} \gamma_{2}}^{(0)}\right\rangle}{z_{3}}\left(\frac{\Delta_{0,1}^{1,1}+\Delta_{2,1}^{1,1}+x_{1}}{x_{1}^{3}}-\frac{\Delta_{0,1}^{2,1}+\Delta_{0,1}^{3,1}}{\left(1-x_{1}\right) x_{2}}\right)+\frac{2 z_{1}\left(\Delta_{0,1}^{1,1}+\Delta_{2,1}^{1,1}+x_{1}\right)}{x_{1}^{3} z_{3}\left(1-z_{1}\right)} \\
& -\frac{\left(1-x_{1}\right)^{2} \bar{\Delta}_{0,1}^{0,3}+2 x_{1}^{3}\left(x_{3}+z_{1}-z_{2}\right)}{x_{1}^{3} x_{2}}+\frac{\left(1-x_{1}\right)\left(z_{2}-\Delta_{0,1}^{1,0}-\bar{\Delta}_{1,3}^{1,1}\right)+2 x_{1}^{3}\left(1-x_{2}\right)}{x_{1}^{3} x_{3}} \\
& +\frac{x_{1}^{2}-2 x_{3}^{2} z_{1}}{x_{1}^{3} x_{2} z_{2}}-\frac{x_{1}^{3}\left(z_{1}+1\right)-4 x_{1}^{2}+6 x_{1} z_{1}\left(z_{1}-1\right)^{-1}+2 z_{1}}{\left(x_{1}-1\right) x_{1}^{3} x_{3} z_{3}} .
\end{aligned}
$$

Note that these coefficients mix both symmetric and antisymmetric contributions, as happened for the component of transcendental weight 1 .

\section{$3.4 \quad g \rightarrow q \bar{q} \gamma$}

Finally, we arrive to the last available configuration in the triple collinear limit with photons. Starting with the leading order, the splitting amplitude associated with the process $g \rightarrow q \bar{q} \gamma$ reads

$$
\mathrm{Sp}_{q_{1} \bar{q}_{2} \gamma_{3}}^{(0)\left(a_{2}, \alpha\right)}=\frac{e_{q} g_{e} g_{\mathrm{S}} \mu^{2 \epsilon} \boldsymbol{T}_{a_{1} a_{2}}^{\alpha}}{s_{123}} \bar{u}\left(p_{1}\right)\left(\frac{\notin\left(p_{3}\right) \not p_{13} \notin(\widetilde{P})}{s_{13}}-\frac{\notin(\widetilde{P}) \not p_{23} \notin\left(p_{3}\right)}{s_{23}}\right) v\left(p_{2}\right),
$$

while the unpolarized splitting function is given by

$$
\begin{aligned}
\left\langle\hat{P}_{q_{1} \bar{q}_{2} \gamma_{3}}^{(0)}\right\rangle= & \frac{e_{q}^{2} g_{e}^{2} g_{\mathrm{S}}^{2}}{2 x_{1} x_{2}}\left[\left(\Delta_{0,1}^{1,0}\right)^{2}+z_{1}^{2}-\Delta\left(\frac{2 z_{2}\left(\Delta_{0,1}^{1,0}+1\right)+\Delta_{0,3}^{3,0}}{1-\Delta}+\frac{\left(1-x_{3}\right)^{2}}{2}\right)\right] \\
& +(1 \leftrightarrow 2),
\end{aligned}
$$



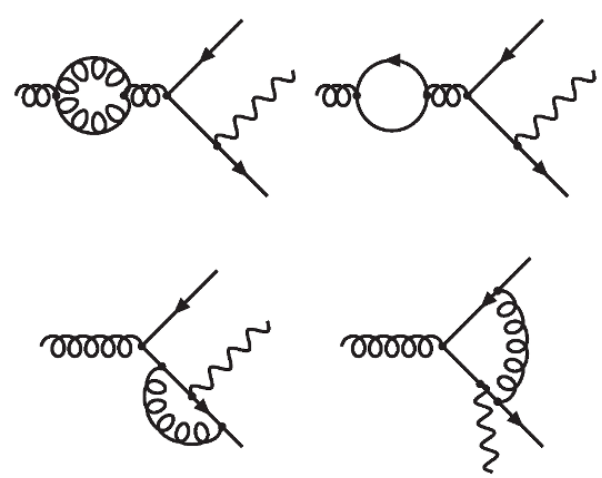
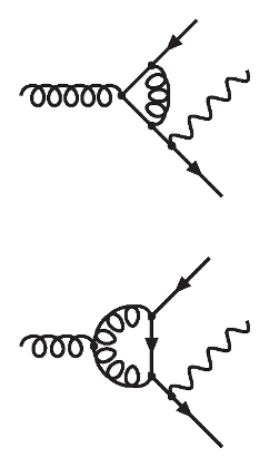
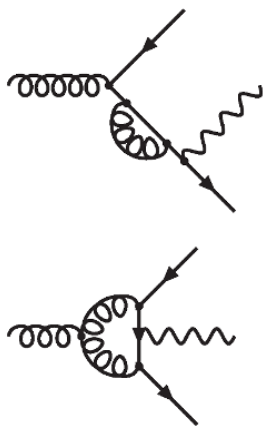

Figure 4. Representative diagrams contributing to the $g \rightarrow q \bar{q} \gamma$ splitting amplitude.

with $\Delta=\delta \epsilon$, as defined for $q \rightarrow q \gamma \gamma$ splitting. We can appreciate that this expression is totally symmetric when interchanging particles 1 and 2 . At amplitude level, there is an additional minus sign coming from the change $e_{q} \rightarrow e_{\bar{q}}=-e_{q}$. On the other hand, it is useful to define

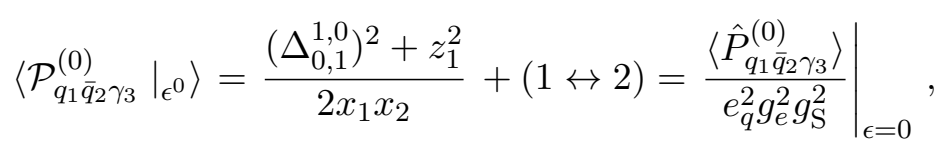

because this expression will allow to simplify NLO results.

Before presenting the results, let's compare this process with $q \rightarrow q g \gamma$. The particles involved are the same, but we exchange the parent quark with a final state gluon. The corresponding Feynman diagrams are shown in figure 4. Excluding self-energy corrections to the parent parton, the other diagrams are in one-to-one correspondence in both processes. Moreover, both sets of diagrams are related by the exchange $P \leftrightarrow 2$. But the parent parton is an off-shell particle, and there are differences if process starts with a gluon or a quark. So, we do not expect to have any crossing transformation relating $\left\langle\hat{P}_{q_{1} \bar{q}_{2} \gamma_{3}}\right\rangle$ and $\left\langle\hat{P}_{q_{1} g_{2} \gamma_{3}}\right\rangle$ beyond tree-level. We will show a conclusive counterexample in the next section.

The IR divergent structure of this splitting is given by

$$
\begin{aligned}
I_{q_{1} \bar{q}_{2} \gamma_{3}}^{(1)}\left(p_{1}, p_{2}, p_{3} ; \widetilde{P}\right)= & \frac{c_{\Gamma} g_{\mathrm{S}}^{2}}{\epsilon^{2}}\left(\frac{-s_{123}-\imath 0}{\mu^{2}}\right)^{-\epsilon}\left[C_{A}\left(2-z_{1}^{-\epsilon}-z_{2}^{-\epsilon}+x_{3}^{-\epsilon}\right)\right. \\
& \left.-2 C_{F} x_{3}^{-\epsilon}-\epsilon\left(2 \gamma_{q}-\gamma_{g}-\frac{\beta_{0}}{2}\right)\right],
\end{aligned}
$$

according to Catani's formula and it agrees with our results.

To express the results in a compact way, we decompose it according to the color structure. So, the finite contribution to the unpolarized splitting function is expanded as

$$
\left\langle\hat{P}_{q_{1} \bar{q}_{2} \gamma_{3}}^{(1) \text { fin. }}\right\rangle=c^{q \bar{q} \gamma}\left[N_{f}\left\langle\hat{P}_{q_{1} \bar{q}_{2} \gamma_{3}}^{\left(1, N_{f}\right) \text { fin. }}\right\rangle+D_{A}\left\langle\hat{P}_{q_{1} \bar{q}_{2} \gamma_{3}}^{\left(1, D_{A}\right) \text { fin. }}\right\rangle+C_{A}\left\langle\hat{P}_{q_{1} \bar{q}_{2} \gamma_{3}}^{\left(1, C_{A}\right) \text { fin. }}\right\rangle+(1 \leftrightarrow 2)\right],
$$

with the global prefactor

$$
c^{q \bar{q} \gamma}=\frac{e_{q}^{2} g_{e}^{2} g_{\mathrm{S}}^{4}}{4(1-\epsilon)}
$$


and

$$
\begin{aligned}
& \left\langle\hat{P}_{q_{1} \bar{q}_{2} \gamma_{3}}^{\left(1, N_{f}\right) \text { fin. }}\right\rangle=C^{\left(0, N_{f}\right)} \\
& \left\langle\hat{P}_{q_{1} \bar{q}_{2} \gamma_{3}}^{\left(1, D_{A}\right) \text { fin. }}\right\rangle=C^{\left(0, D_{A}\right)}+\sum_{i=1}^{2} C_{i}^{\left(1, D_{A}\right)} F_{i}^{(1)}+C_{1}^{\left(2, D_{A}\right)} F_{1}^{\left(2, D_{A}\right)} \\
& \left\langle\hat{P}_{q_{1} \bar{q}_{2} \gamma_{3}}^{\left(1, C_{A}\right) \text { fin. }}\right\rangle=C^{\left(0, C_{A}\right)}+C_{1}^{\left(1, C_{A}\right)} F_{1}^{(1)}+\sum_{j=1}^{4} C_{j}^{\left(2, C_{A}\right)} F_{j}^{\left(2, C_{A}\right)},
\end{aligned}
$$

where we classify each contribution according to its transcendental weight. We can appreciate that the part proportional to $N_{f}$ is purely rational. Since it is originated from diagrams which contain fermionic loops, only standard bubbles (i.e. without the extra LCG propagator) can contribute to $N_{f}$ terms. But these bubbles have single $\epsilon$ poles which are completely absorbed into $\boldsymbol{I}_{q_{1} \bar{q}_{2} \gamma_{3}}^{(1)}\left(p_{1}, p_{2}, p_{3} ; \widetilde{P}\right)$, including also the corresponding logarithms. So, only the rational terms proportional to $N_{f}$ survive after the subtraction procedure.

Now let's show the explicit results. Starting with the rational terms, we have

$$
\begin{aligned}
C^{\left(0, N_{f}\right)}= & -\frac{20}{9}\left\langle\left.\mathcal{P}_{q_{1} \bar{q}_{2} \gamma_{3}}^{(0)}\right|_{\epsilon^{0}}\right\rangle \\
C^{\left(0, C_{A}\right)}= & \frac{71}{9}\left\langle\left.\mathcal{P}_{q_{1} \bar{q}_{2} \gamma_{3}}^{(0)}\right|_{\epsilon^{0}}\right\rangle+\frac{z_{1}}{x_{3}}\left(\frac{x_{3}\left(1-2 z_{3}\right)+z_{1}}{x_{1}-1}+\frac{\left(x_{3}+1\right)\left(x_{3}-z_{1}\right)}{x_{2}\left(x_{3}-1\right)}\right) \\
& +\frac{x_{3}\left(z_{1}^{2}-z_{1}+1\right)\left(x_{3}-2 x_{1}+1\right)}{\left(1-x_{1}\right) x_{1}\left(1-x_{3}\right)} \\
C^{\left(0, D_{A}\right)}= & \left(x_{1} x_{2}-z_{1} z_{2}-\Delta_{0,1}^{1,0} \Delta_{0,2}^{2,0}\right)\left(\frac{2-2 x_{1}\left(x_{2}+1\right)}{x_{1} x_{2}\left(1-x_{3}\right)}-\frac{1}{1-x_{1}}\right) \\
& -\frac{2 z_{1} \Delta_{0,1}^{1,0}}{x_{1} x_{2}}\left(\frac{\left(x_{2}+1\right)\left(1-x_{2}\right)^{2}}{\left(1-x_{1}\right)\left(1-x_{3}\right)}+\frac{x_{3}-x_{1} x_{2}}{1-x_{3}}+\frac{\left(3-x_{2}\right) x_{2}-x_{1}\left(x_{2}+1\right)}{2\left(1-x_{1}\right)}+8\right) \\
& -\frac{8\left(1-x_{1}\right)^{2}}{x_{1} x_{2}}-\frac{1-x_{1}}{x_{1}},
\end{aligned}
$$

where we can appreciate that the expressions are symmetric under the transformation $1 \leftrightarrow 2$.

The basis for the contribution of transcendental weight 1 is given by

$$
\begin{aligned}
& F_{1}^{(1)}=\log \left(x_{1}\right), \\
& F_{2}^{(1)}=\log \left(x_{3}\right),
\end{aligned}
$$

and the associated coefficients are

$$
\begin{aligned}
C_{1}^{\left(1, D_{A}\right)}= & \frac{x_{1} x_{2}-z_{1} z_{2}-\Delta_{0,1}^{1,0} \Delta_{0,2}^{2,0}}{\left(1-x_{1}\right) x_{1}}\left(\frac{x_{2}+2 x_{3}}{x_{2}^{2}}-\frac{x_{1} x_{2}+2 x_{3}}{\left(1-x_{1}\right) x_{2}}-\frac{1+x_{1}}{1-x_{1}}\right)-\frac{z_{2}\left(2 x_{3}-x_{2}\right) \Delta_{0,2}^{2,0}}{x_{1} x_{2}^{2}} \\
& -\frac{\left(1-x_{2}\right)^{2} z_{1}\left(2\left(1-x_{1}\right)+x_{2}\right) \Delta_{0,1}^{1,0}}{\left(1-x_{1}\right)^{2} x_{1} x_{2}^{2}}+\frac{\left(1-x_{2}\right)\left(x_{2}-2 x_{3}\right)}{\left(1-x_{1}\right) x_{2}}, \\
C_{2}^{\left(1, D_{A}\right)}= & \frac{2\left(2 x_{1}\left(z_{2}-1-\Delta_{0,2}^{2,0}\left(x_{1} x_{2}+1\right)\right)-\left(\Delta_{1,2}^{0,3}\right)^{2}-2 x_{2}\left(z_{1}+2 z_{2}-3\right)\left(x_{1} x_{2}+2 z_{3}\right)-x_{2} z_{3}\right)}{x_{2}^{2}\left(1-x_{3}\right)^{2}}
\end{aligned}
$$




$$
\begin{aligned}
& -\frac{2\left(x_{1}^{2}\left(2 z_{1}\left(z_{2}-3\right)+\left(4 z_{2}-13\right) z_{2}+7\right)+2 z_{3}^{2}\right)}{x_{1} x_{2}\left(1-x_{3}\right)^{2}} \\
& -\frac{2\left(2 x_{1} x_{2}+\left(z_{1}-15\right) z_{1}+7\right)}{\left(1-x_{3}\right)^{2}}
\end{aligned}
$$

for the Abelian part, and

$$
\begin{aligned}
C_{1}^{\left(1, C_{A}\right)}= & \frac{z_{2}\left(2\left(x_{2}-2\right) z_{1}-9 x_{2}+10\right)+\left(x_{2}-1\right)\left(2 x_{2}\left(z_{1}-2\right)-3 z_{1}+4\right)-6 z_{2}^{2}}{\left(1-x_{1}\right)^{2} x_{2}} \\
& +\frac{x_{1}^{2}\left(3\left(\Delta_{0,2}^{2,0}\right)^{2}-\Delta_{2,2}^{0,0}+\bar{\Delta}_{2,2}^{0,2}\right)+\Delta_{2,1}^{0,3}\left(\Delta_{2,1}^{0,3}-\Delta_{0,2}^{2,0}-z_{2}\right)}{\left(1-x_{1}\right)^{2} x_{1} x_{2}}
\end{aligned}
$$

for the $C_{A}$ terms.

Finally, if we go to transcendental weight 2 , we can write the term proportional to $D_{A}$ making use of

$$
\begin{aligned}
F_{1}^{\left(2, D_{A}\right)}= & \mathcal{R}\left(x_{1}, x_{3}\right), \\
C_{1}^{\left(2, D_{A}\right)}= & \frac{2\left(x_{2}\left(x_{3} \Delta_{3,2}^{0,1}+\Delta_{0,3}^{3,0}\left(\Delta_{2,3}^{0,1}+z_{1}\right)+x_{2}^{3}+2 x_{2} x_{3} z_{1}\right)+\left(\Delta_{3,2}^{0,1}\right)^{2}\right)}{x_{1} x_{2}^{3}} \\
& -\frac{4 \Delta_{0,1}^{1,0}\left(x_{3}-z_{1}\right)}{x_{1} x_{2}},
\end{aligned}
$$

while for the component proportional to $C_{A}$ we require

$$
\begin{aligned}
F_{1}^{\left(2, C_{A}\right)}= & \frac{\pi^{2}}{6}-2 \operatorname{Li}_{2}\left(1-\frac{x_{1}}{1-z_{1}}\right)-2 \operatorname{Li}_{2}\left(1-\frac{z_{2}}{1-z_{1}}\right)+2 \operatorname{Li}_{2}\left(1-z_{1}\right) \\
& +2 \log \left(x_{2}\right) \log \left(1-z_{1}\right)+(1 \leftrightarrow 2), \\
F_{2}^{\left(2, C_{A}\right)}= & \log \left(x_{1}\right) \log \left(x_{2}\right), \\
F_{3}^{\left(2, C_{A}\right)}= & \frac{\pi^{2}}{4}-\operatorname{Li}_{2}\left(1-x_{1}\right)-\log \left(x_{1}\right) \log \left(z_{1}\right)+(1 \leftrightarrow 2), \\
F_{4}^{\left(2, C_{A}\right)}= & \log \left(\frac{x_{1}}{1-z_{1}}\right) \log \left(\frac{1-z_{1}}{z_{1} z_{2}}\right)-\log \left(x_{2}\right) \log \left(1-z_{1}\right),
\end{aligned}
$$

with the associated coefficients

$$
\begin{aligned}
C_{1}^{\left(2, C_{A}\right)}= & \frac{z_{1}^{2}}{x_{1} x_{2}}, \\
C_{2}^{\left(2, C_{A}\right)}= & \frac{z_{2}-x_{1} \Delta_{0,2}^{2,0}-x_{1}}{x_{1} \Delta_{0,1}^{1,0}}+\frac{1-x_{2}}{x_{1}} \\
C_{3}^{\left(2, C_{A}\right)}= & 2 \frac{2\left(1-z_{1}\right) z_{1}-x_{1}^{2}+3 x_{1}-1}{x_{1} x_{2}}-\frac{2\left(2 z_{1}+1\right)}{x_{2}}, \\
C_{4}^{\left(2, C_{A}\right)}= & \frac{\left(\left(1-z_{2}\right)^{2}+z_{2}^{2}\right) \Delta_{1,2}^{2,2}}{x_{1} x_{2} z_{3}}+\frac{z_{2}-x_{1}\left(\Delta_{0,2}^{2,0}+1\right)}{x_{1} \Delta_{0,1}^{1,0}}+\frac{x_{2}+2\left(2 z_{2}^{2}+z_{2}-1\right)}{x_{1}} \\
& +\frac{z_{3}\left(3\left(x_{2}+2\right) z_{2}-4-x_{1}\left(1-z_{2}\right)\right)}{x_{1} x_{2}}+\frac{3-2\left(x_{1}+3\right)\left(1-z_{2}\right) z_{2}}{x_{1} x_{2}} \\
& +\frac{\left(x_{2}+3\right) z_{3}^{2}}{x_{1} x_{2}} .
\end{aligned}
$$




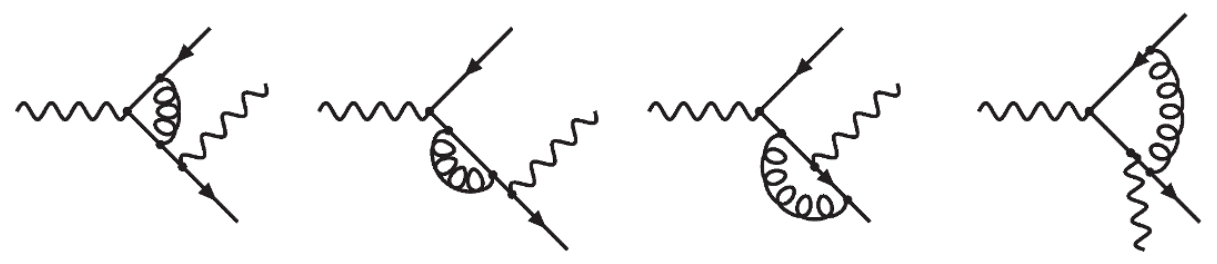

Figure 5. Representative diagrams contributing to the $\gamma \rightarrow q \bar{q} \gamma$ splitting amplitude. We neglect self-energy corrections to the parent photon because they contribute at a higher QED order.

It is interesting to notice that these expressions are very compact, especially if we compare them with the analogous expansion for $q \rightarrow q \gamma \gamma$ and $q \rightarrow q g \gamma$. This fact is related with the presence of a initial-state vector particle, as we discuss in the next section.

\section{Processes started by photons}

Finally, we also considered NLO QCD corrections to splittings functions started by photons. These objects might be relevant to describe the decay of a virtual photon into three on-shell massless particles up to NLO accuracy in QCD. Following the discussion presented in the previous sections, we consider the two photon-started triple splitting processes: $\gamma \rightarrow q \bar{q} \gamma$ and $\gamma \rightarrow q \bar{q} g$. Moreover, let's recall that $\gamma \rightarrow n g$ vanish at tree-level for all $n \in \mathbb{N}$ due to decoupling identities, which implies that $\gamma \rightarrow n g$ must be finite beyond LO.

Besides presenting the results, we take advantage of their simplicity in order to extract some interesting conclusions about their functional structure.

\section{$4.1 \gamma \rightarrow q \bar{q} \gamma$}

Beginning with the LO splitting amplitude, we have

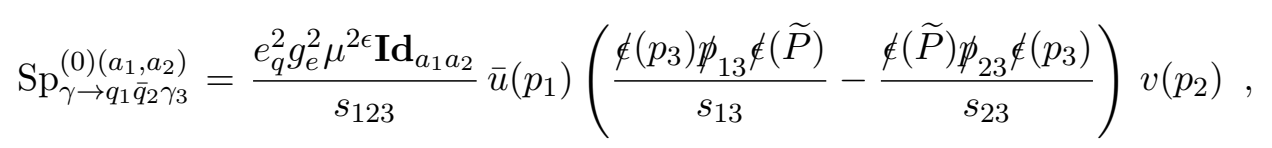

where it is possible to appreciate that this amplitude is just the color stripped contribution to $\boldsymbol{S p}_{q_{1} \bar{q}_{2} \gamma_{3}}^{(0)}$. Explicitly,

$$
\operatorname{Sp}_{q_{1} \bar{q}_{2} \gamma_{3}}^{(0)\left(a_{1}, a_{2} ; \alpha\right)}=\frac{g_{\mathrm{S}}}{g_{e} e_{q}} \boldsymbol{T}_{a_{1} a_{2}}^{\alpha} \operatorname{Sp}_{\gamma \rightarrow q_{1} \bar{q}_{2} \gamma_{3}}^{(0)\left(a_{1}, a_{2}\right)}
$$

which trivially implies that the unpolarized splitting function is given by

$$
\left\langle\hat{P}_{\gamma \rightarrow q_{1} \bar{q}_{2} \gamma_{3}}^{(0)}\right\rangle=\frac{C_{A} g_{e}^{2} e_{q}^{2}}{C_{F} g_{\mathrm{S}}^{2}}\left\langle\hat{P}_{q_{1} \bar{q}_{2} \gamma_{3}}^{(0)}\right\rangle,
$$

using results from the previous section. Note that symmetry under the exchange $1 \leftrightarrow 2$ is obviously inherited.

When we considered $g \rightarrow q \bar{q} \gamma$ in section 3, we arranged the different contributions to the NLO splitting function in order to identify Abelian and non-Abelian terms. We saw that purely Abelian terms were given by compact expressions which can be written in 
terms of logarithms and just one weight 2 function. Since $\gamma \rightarrow q \bar{q} \gamma$ is proportional to the Abelian part, its splitting function is extremely simple. In figure 5 we show all the Feynman diagrams required in the computation. Notice that self-energy corrections to the incoming photon are not taken into account because they contribute to higher-orders in QED.

Following the same recipe for the other processes, we start studying the IR divergent structure. According to Catani's formula, we have

$$
I_{\gamma \rightarrow q_{1} \bar{q}_{2} \gamma_{3}}^{(1)}\left(p_{1}, p_{2}, p_{3} ; \widetilde{P}\right)=\frac{c_{\Gamma} g_{\mathrm{S}}^{2}}{\epsilon^{2}}\left(\frac{-s_{123}-\imath 0}{\mu^{2}}\right)^{-\epsilon}\left[-2 C_{F} x_{3}^{-\epsilon}-2 \epsilon \gamma_{q}\right]
$$

and we found a complete agreement with our results. On the other hand, after performing the full computation we notice that the NLO splitting function can be expressed as

$$
\left\langle\hat{P}_{\gamma \rightarrow q_{1} \bar{q}_{2} \gamma_{3}}^{(1) \text { fin. }}\right\rangle=2 C_{F} c^{\gamma \rightarrow q \bar{q} \gamma}\left[\left\langle\hat{P}_{q_{1} \overline{q_{2}} \gamma_{3}}^{\left(1, D_{A}\right) \text { fin. }}\right\rangle+(1 \leftrightarrow 2)\right],
$$

as it was expected based in a naive Feynman diagram comparison between these processes. Here we introduced the global prefactor

$$
c^{\gamma \rightarrow q \bar{q} \gamma}=\frac{C_{A} e_{q}^{4} g_{e}^{4} g_{\mathrm{S}}^{2}}{2(1-\epsilon)},
$$

in order to simplify the notation.

To conclude this section, let's make a comment about crossing identities for splitting functions (and amplitudes). In section 3, we presented $q \rightarrow q \gamma \gamma$ and the simplified weight 2 contribution to the NLO correction could be expressed in terms of 11 functions. However $\left.\left\langle\hat{P}_{\gamma \rightarrow q_{1} \bar{q}_{2} \gamma_{3}}^{(1) \text { fin. }}\right\rangle\right|_{w=2}$ is proportional to just one of these functions. Since crossing relations are (rational) variable transformations, they can not modify the dimension of the space of functions which span the transcendental weight 2 contribution. We can explain this situation reasoning as follows. To obtain $\gamma \rightarrow q \bar{q} \gamma$ we need to perform the exchange $2 \leftrightarrow P$. This implies changing the parent parton, which is a distinguished particle from a kinematical point of view (i.e. it is slightly off-shell and this fact involves that self-energy corrections can not be neglected). To be more explicit, in $q \rightarrow q \gamma \gamma$ the parent quark is offshell and outgoing photons are on-shell, but in $\gamma \rightarrow q \bar{q} \gamma$ we have one off-shell photon and the quark that has been moved to the final state loses its virtuality. So, strictly speaking, kinematics forces us to consider that the particle content of these splittings is not the same.

\section{$4.2 \gamma \rightarrow q \bar{q} g$}

Finally, we arrive to the last non-trivial triple splitting process with photons: $\gamma \rightarrow q \bar{q} g$. The corresponding Feynman diagrams at NLO are shown in figure 6 .

The LO splitting amplitude can be written as

$$
\begin{aligned}
\operatorname{Sp}_{\gamma \rightarrow q_{1} \bar{q}_{2} g_{3}}^{(0)\left(a_{1}, a_{2}, \alpha_{3}\right)} & =\frac{e_{q} g_{e} g_{\mathrm{S}} \mu^{2 \epsilon} \boldsymbol{T}_{a_{1} a_{2}}^{\alpha_{3}}}{s_{123}} \bar{u}\left(p_{1}\right)\left(\frac{\notin\left(p_{3}\right) \not p_{13} \notin(\widetilde{P})}{s_{13}}-\frac{\notin(\widetilde{P}) \not p_{23} \notin\left(p_{3}\right)}{s_{23}}\right) v\left(p_{2}\right) \\
& =\frac{g_{\mathrm{S}}}{g_{e} e_{q}} \boldsymbol{T}_{a_{1} a_{2}}^{\alpha_{3}} \operatorname{Sp}_{\gamma \rightarrow q_{1} \bar{q}_{2} \gamma_{3}}^{(0)\left(a_{1}, a_{2}\right)}
\end{aligned}
$$




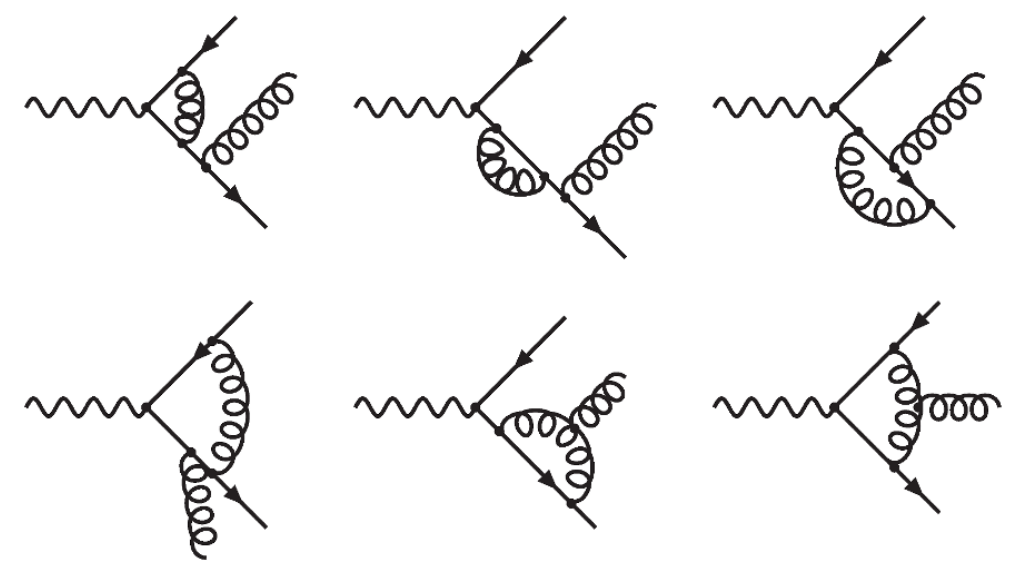

Figure 6. Representative diagrams contributing to the $\gamma \rightarrow q \bar{q} g$ splitting amplitude.

where we used previous results to obtain the last line. In fact, using that relation with the $\gamma \rightarrow q \bar{q} \gamma$ splitting, the corresponding splitting function at LO turns out to be

$$
\left\langle\hat{P}_{\gamma \rightarrow q_{1} \bar{q}_{2} g_{3}}^{(0)}\right\rangle=\frac{g_{\mathrm{S}}^{2} C_{F}}{g_{e}^{2} e_{q}^{2}}\left\langle\hat{P}_{\gamma \rightarrow q_{1} \bar{q}_{2} \gamma_{3}}^{(0)}\right\rangle=2 C_{A} C_{F}\left\langle\hat{P}_{q_{1} \bar{q}_{2} \gamma_{3}}^{(0)}\right\rangle,
$$

which can easily be related with different splitting functions interchanging photons and gluons. Note that we are not changing kinematics but only the color structure.

In order to express results for the NLO correction, we follow the same strategy used with $g \rightarrow q \bar{q} \gamma$. Since there are contributions proportional to both $C_{A}$ and $D_{A}=2 C_{F}-C_{A}$, we treat them separately. The contribution proportional to $D_{A}$ is just the one coming from $\left\langle\hat{P}_{\gamma \rightarrow q_{1} \bar{q}_{2} \gamma_{3}}^{(1)}\right\rangle$ (with a factor 2 of difference due to the definition of $D_{A}$ ). To check the global IR divergent structure, we extract the $\epsilon$ poles from the splitting function and compare them with the expression provided by Catani's formula, i.e.

$$
\begin{aligned}
I_{\gamma \rightarrow q_{1} \bar{q}_{2} g_{3}}^{(1)}\left(p_{1}, p_{2}, p_{3} ; \widetilde{P}\right)= & I_{\gamma \rightarrow q_{1} \bar{q}_{2} \gamma_{3}}^{(1)}\left(p_{1}, p_{2}, p_{3} ; \widetilde{P}\right) \\
& +\frac{c_{\Gamma} g_{\mathrm{S}}^{2} C_{A}}{\epsilon^{2}}\left(\frac{-s_{123}-\imath 0}{\mu^{2}}\right)^{-\epsilon}\left(x_{3}^{-\epsilon}-x_{1}^{-\epsilon}-x_{2}^{-\epsilon}\right),
\end{aligned}
$$

finding a complete agreement. Note that we used the operator $\boldsymbol{I}_{\gamma \rightarrow q_{1} \bar{q}_{2} \gamma_{3}}^{(1)}$ from eq. (4.4) to simplify the results. Moreover, following this idea, we write the NLO correction to the splitting function as

$$
\left\langle\hat{P}_{\gamma \rightarrow q_{1} \bar{q}_{2} g_{3}}^{(1)}\right\rangle=D_{A}\left\langle\hat{P}_{\gamma \rightarrow q_{1} \bar{q}_{2} \gamma_{3}}^{\left(1, D_{1}\right)}\right\rangle+C_{A}\left\langle\hat{P}_{\gamma \rightarrow q_{1} \bar{q}_{2} g_{3}}^{\left(1, C_{g_{3}}\right.}\right\rangle+\text { c.c. },
$$

with

$$
\begin{aligned}
\left\langle\hat{P}_{\gamma \rightarrow q_{1} \bar{q}_{2} g_{3}}^{\left(1, D_{A}\right)}\right\rangle & =\frac{1}{2 C_{F}}\left[\operatorname{Re}\left(I_{\gamma \rightarrow q_{1} \bar{q}_{2} \gamma_{3}}^{(1)}\left(p_{1}, p_{2}, p_{3} ; \widetilde{P}\right)\right)\left\langle\hat{P}_{\gamma \rightarrow q_{1} \bar{q}_{2} g_{3}}^{(0)}\right\rangle+\frac{c^{\gamma \rightarrow q \bar{q} g}}{c^{\gamma \rightarrow q \bar{q} \gamma}}\left\langle\hat{P}_{\gamma \rightarrow q_{1} \bar{q}_{2} \gamma_{3}}^{(1) \text { fin. }}\right\rangle\right] \\
& =\frac{g_{\mathrm{S}}^{2}}{2 e_{q}^{2} g_{e}^{2}}\left[\operatorname{Re}\left(I_{\gamma \rightarrow q_{1} \bar{q}_{2} \gamma_{3}}^{(1)}\left(p_{1}, p_{2}, p_{3} ; \widetilde{P}\right)\right)\left\langle\hat{P}_{\gamma \rightarrow q_{1} \bar{q}_{2} \gamma_{3}}^{(0)}\right\rangle+\left\langle\hat{P}_{\gamma \rightarrow q_{1} \bar{q}_{2} \gamma_{3}}^{(1)}\right\rangle\right]
\end{aligned}
$$


where we introduced the global prefactor

$$
c^{\gamma \rightarrow q \bar{q} g}=\frac{C_{A} C_{F} e_{q}^{2} g_{e}^{2} g_{\mathrm{S}}^{4}}{2(1-\epsilon)} .
$$

To treat the finite contribution, we express it as

$$
\left\langle\hat{P}_{\gamma \rightarrow q_{1} \bar{q}_{2} g_{3}}^{(1) \text { fin. }}\right\rangle=c^{\gamma \rightarrow q \bar{q} g}\left[D_{A}\left\langle\hat{P}_{\gamma \rightarrow q_{1} \bar{q}_{2} g_{3}}^{\left(1, D_{A}\right) \text { fin. }}\right\rangle+C_{A}\left\langle\hat{P}_{\gamma \rightarrow q_{1} \bar{q}_{2} g_{3}}^{\left(1, C_{A}\right) \text { fin. }}\right\rangle\right],
$$

where the finite Abelian contribution is given by

$$
\left\langle\hat{P}_{\gamma \rightarrow q_{1} \bar{q}_{2} g_{3}}^{\left(1, D_{A}\right) \text { fin. }}\right\rangle=\frac{g_{\mathrm{S}}^{2}}{2 e_{q}^{2} g_{e}^{2}}\left\langle\hat{P}_{\gamma \rightarrow q_{1} \bar{q}_{2} \gamma_{3}}^{(1) \text { fin. }}\right\rangle=c^{\gamma \rightarrow q \bar{q} g}\left\langle\hat{P}_{q_{1} \bar{q}_{2} \gamma_{3}}^{\left(1, D_{A}\right) \text { fin. }}\right\rangle .
$$

The novel contributions originated in the non-Abelian part are contained inside $\left\langle\hat{P}_{\gamma \rightarrow q_{1} q_{2} g_{3}}^{\left(1, C_{A}\right){ }_{i n}}\right\rangle$. Classifying these terms according to its transcendental weight, we get

$$
\left\langle\hat{P}_{\gamma \rightarrow q_{1} \bar{q}_{2} g_{3}}^{\left(1, C_{A}\right) \text { fin. }}\right\rangle=C^{\left(0, C_{A}\right)}+C^{\left(1, C_{A}\right)} F^{(1)}+C^{\left(2, C_{A}\right)} F^{\left(2, C_{A}\right)}+(1 \leftrightarrow 2),
$$

where the rational coefficient is given by

$$
\begin{aligned}
C^{\left(0, C_{A}\right)}= & \frac{16-7 x_{2}-2 z_{1} z_{2}+\left(1-z_{1}\right)^{2}-15 z_{2}}{x_{1}}-\frac{z_{1}^{2}}{\left(1-x_{1}\right) x_{2}}-8 \frac{z_{1}^{2}+\left(1-z_{1}\right)^{2}}{x_{1} x_{2}} \\
& +\frac{2 z_{1}\left(1-z_{3}\right)-x_{2}\left(1-z_{1}\right)^{2}-\left(x_{2}+1\right) z_{1}}{\left(1-x_{1}\right) x_{1}}
\end{aligned}
$$

and

$$
\begin{aligned}
F^{(1)} & =\log \left(x_{1}\right), \\
F^{\left(2, C_{A}\right)} & =\mathcal{R}\left(x_{1}, x_{2}\right),
\end{aligned}
$$

are the functions which expands the spaces of transcendentality 1 and 2 , respectively. It is crucial to appreciate that $F^{\left(2, C_{A}\right)}$ is the same function involved in the $\gamma \rightarrow q \bar{q} \gamma$ (aside from a permutation of the kinematical variables). We will return to this point in the next subsection. Finally, in order to get the full NLO correction to this splitting function, the coefficients listed in eq. (4.15) are given by

$$
\begin{aligned}
C^{\left(1, C_{A}\right)}= & \frac{z_{2}\left(x_{2}\left(4 x_{1} z_{1}+x_{1}-1\right)+2 x_{3} z_{1}\right)+x_{2}\left(x_{1}\left(\left(x_{2}-1\right) z_{1}+x_{2}-3\right)-2 x_{2}+3\right)}{\left(x_{1}-1\right)^{2} x_{1} x_{2}} \\
& +\frac{3 x_{2}^{2}+5 x_{2}\left(z_{2}-1\right)+3 z_{2}^{2}-4 z_{2}+1}{x_{1} x_{2}}-\frac{\left(1-x_{2}\right)^{2} z_{1}{ }^{2}}{\left(1-x_{1}\right)^{2} x_{1} x_{2}} \\
C^{\left(2, C_{A}\right)=} & \left\langle\mathcal{P}_{q_{1} \bar{q}_{2} \gamma_{3}}^{(0)} \mid \epsilon^{0}\right\rangle
\end{aligned}
$$

Again, it is possible to appreciate that this splitting can not easily be related to $g \rightarrow q \bar{q} \gamma$. In particular, in section 3 we showed that 7 functions were required to expand the component of transcendental weight 2 for $\left\langle\hat{P}_{q_{1} \bar{q}_{2} \gamma_{3}}^{(1) \text { fin. }}\right\rangle$. However, $\left\langle\hat{P}_{\gamma \rightarrow q_{1}}^{(1) \text { fin. }} g_{3}\right\rangle$ involves a 2-dimensional transcendental weight 2 space. 


\subsection{Further discussions on splittings started by photons}

Since the results shown in this section are very simple (compared with those presented in section 3), we can go further and discuss about the structure of triple splittings started by photons.

Let's start with $\gamma \rightarrow q \bar{q} \gamma$. If we perform a direct computation without making an $\epsilon$-expansion, the NLO contribution to the splitting function can be expressed as

$$
\left\langle\hat{P}_{\gamma \rightarrow q_{1} \bar{q}_{2} \gamma_{3}}^{(1)}\right\rangle=A_{1}^{(4)}\left(z_{j}, x_{k} ; \epsilon\right) I_{1}^{(4)}+\sum_{i=1}^{3} A_{i}^{(2)}\left(z_{j}, x_{k} ; \epsilon\right) I_{i}^{(2)}+(1 \leftrightarrow 2),
$$

where coupling constants and color factors are absorbed inside the coefficients $A$. Here $A_{i}^{(4)}$ and $A_{i}^{(2)}$ are rational functions of the kinematical variables $\left\{x_{i}, z_{j}\right\}$ and $\epsilon$. Branch-cuts are defined by the Feynman integrals $I_{1}^{(4)}$ (box) and $I_{i}^{(2)}$ (bubbles). Moreover, using $d=4-2 \epsilon$, the explicit expression for the box integral is

$$
\begin{aligned}
I_{1}^{(4)}= & \int_{q} \frac{\mu^{2 \epsilon}}{q^{2}\left(q+p_{1}\right)^{2}\left(q-p_{2}\right)^{2}\left(q-p_{23}\right)^{2}}=\frac{2 c_{\Gamma}}{\epsilon^{2} x_{1} x_{3} s_{123}^{2}}\left(\frac{-s_{123}-\imath 0}{\mu^{2}}\right)^{-\epsilon} \\
& \times\left[x_{1}^{-\epsilon}{ }_{2} F_{1}\left(1,-\epsilon ; 1-\epsilon ;-\frac{x_{2}}{x_{3}}\right)+x_{3}^{-\epsilon}{ }_{2} F_{1}\left(1,-\epsilon ; 1-\epsilon ;-\frac{x_{2}}{x_{1}}\right)\right. \\
& \left.-{ }_{2} F_{1}\left(1,-\epsilon ; 1-\epsilon ;-\frac{x_{2}}{x_{1} x_{3}}\right)\right],
\end{aligned}
$$

while bubbles are given by

$$
\begin{aligned}
& I_{1}^{(2)}=\int_{q} \frac{\mu^{2 \epsilon}}{q^{2}\left(q-p_{123}\right)^{2}}=\frac{c_{\Gamma}}{\epsilon(1-2 \epsilon)}\left(\frac{-s_{123}-\imath 0}{\mu^{2}}\right)^{-\epsilon}, \\
& I_{2}^{(2)}=\int_{q} \frac{\mu^{2 \epsilon}}{q^{2}\left(q-p_{23}\right)^{2}}=\frac{c_{\Gamma} x_{1}^{-\epsilon}}{\epsilon(1-2 \epsilon)}\left(\frac{-s_{123}-\imath 0}{\mu^{2}}\right)^{-\epsilon}, \\
& I_{3}^{(2)}=\int_{q} \frac{\mu^{2 \epsilon}}{q^{2}\left(q-p_{12}\right)^{2}}=\frac{c_{\Gamma} x_{3}^{-\epsilon}}{\epsilon(1-2 \epsilon)}\left(\frac{-s_{123}-\imath 0}{\mu^{2}}\right)^{-\epsilon},
\end{aligned}
$$

where we defined

$$
\int_{q}=-\imath \int \frac{d^{d} q}{(2 \pi)^{d}}
$$

in order to simplify the notation. We can appreciate that these integrals are known to all orders in $\epsilon$ which implies that the same can be said about $\left\langle\hat{P}_{\gamma \rightarrow q_{1} \bar{q}_{2} \gamma_{3}}^{(1)}\right\rangle$.

If we study the integrals involved in NLO corrections to $\gamma \rightarrow q \bar{q} g$, we realize that an analogous situation takes place. In other words, it turns out to be possible to write

$$
\begin{aligned}
\left\langle\hat{P}_{\gamma \rightarrow q_{1} \bar{q}_{2} g_{3}}^{(1)}\right\rangle= & A_{1}^{(4)}\left(z_{j}, x_{k} ; \epsilon\right) I_{1}^{(4)}+A_{2}^{(4)}\left(z_{j}, x_{k} ; \epsilon\right) I_{2}^{(4)} \\
& +\sum_{i=1}^{3} A_{i}^{(2)}\left(z_{j}, x_{k} ; \epsilon\right) I_{i}^{(2)}+(1 \leftrightarrow 2),
\end{aligned}
$$

where

$$
I_{2}^{(4)}=\int_{q} \frac{\mu^{2 \epsilon}}{q^{2}\left(q+p_{2}\right)^{2}\left(q-p_{3}\right)^{2}\left(q-p_{13}\right)^{2}}=\frac{2 c_{\Gamma}}{\epsilon^{2} x_{1} x_{2} s_{123}^{2}}\left(\frac{-s_{123}-\imath 0}{\mu^{2}}\right)^{-\epsilon}
$$




$$
\begin{aligned}
\times & {\left[x_{2}^{-\epsilon}{ }_{2} F_{1}\left(1,-\epsilon ; 1-\epsilon ;-\frac{x_{3}}{x_{1}}\right)+x_{1}^{-\epsilon}{ }_{2} F_{1}\left(1,-\epsilon ; 1-\epsilon ;-\frac{x_{3}}{x_{2}}\right)\right.} \\
& \left.-{ }_{2} F_{1}\left(1,-\epsilon ; 1-\epsilon ;-\frac{x_{3}}{x_{1} x_{2}}\right)\right]=\left.I_{1}^{(4)}\right|_{2 \leftrightarrow 3},
\end{aligned}
$$

is just a standard one-mass box integral. The corresponding coefficients can be computed to all orders in $\epsilon$, but their explicit expressions are a bit cumbersome.

After motivating the results for $\left\langle\hat{P}_{\gamma \rightarrow q_{1} \bar{q}_{2} \gamma_{3}}^{(1)}\right\rangle$ and $\left\langle\hat{P}_{\gamma \rightarrow q_{1} \bar{q}_{2} g_{3}}^{(1)}\right\rangle$, it is interesting to appreciate that

$$
I_{1}^{(4)}=\frac{2 c_{\Gamma}}{x_{1} x_{3} s_{123}^{2}}\left(\frac{-s_{123}-\imath 0}{\mu^{2}}\right)^{-\epsilon}\left[\frac{1}{\epsilon^{2}}-\frac{\log \left(x_{1} x_{3}\right)}{\epsilon}+\frac{\log ^{2}\left(x_{1}\right)+\log ^{2}\left(x_{3}\right)}{2}-\mathcal{R}\left(x_{1}, x_{3}\right)\right],
$$

which explains the origin of the function $\mathcal{R}$. In other words, it originates from the hypergeometric functions involved in the standard scalar boxes (extracting squared logarithms introduced by standard bubbles). But the important fact is that this one appears in all triple-collinear splitting functions with photons and also in the antisymmetric part of $\left\langle\hat{P}_{q_{1} \bar{Q}_{2} Q_{3}}^{(1)}\right\rangle$ (computed in ref. [24]). So, the presence of this function is due to purely kinematical reasons. Another interesting fact is that to span triple collinear splitting functions started by photons, we only used boxes (maximal topological complexity) and bubbles (minimal non-trivial topology): triangles do not contribute to this computation.

There is a last point which deserves to be discussed. We have seen that splittings started by gluons are simpler than quark-started ones. In same sense this sounds reasonable because spinors only satisfy Dirac's equation, while physical gluons should verify transversality and gauge invariance. Explicitly, when we compute splitting amplitudes we start with an off-shell amputated amplitude $\mathcal{A}_{\mathrm{amp}}$ and then we project it over an on-shell spinor or polarization vector, $u(\widetilde{P})$ or $\epsilon(\widetilde{P})$ respectively. But spinors satisfy

$$
\widetilde{P} u(\widetilde{P})=0,
$$

while on-shell massless polarization vectors verify

$$
\begin{gathered}
\widetilde{P} \cdot \epsilon(\widetilde{P})=0, \\
n \cdot \epsilon(\widetilde{P})=0,
\end{gathered}
$$

where $n$ is a null-vector which defines the light-cone gauge. On the other hand, we can write

$$
\begin{aligned}
\boldsymbol{S p}_{q \rightarrow a_{1} \ldots a_{m}} & =\left.\frac{1}{s_{123}} \mathcal{A}_{(\mathrm{amp}, q)}^{(1, i)}\right|_{\mathrm{LCG}} u_{i}(\widetilde{P}), \\
\boldsymbol{S p}_{g \rightarrow a_{1} \ldots a_{m}} & =\left.\frac{1}{s_{123}} \mathcal{A}_{(\mathrm{amp}, g)}^{(1, \mu)}\right|_{\mathrm{LCG}} \epsilon_{\mu}(\widetilde{P}),
\end{aligned}
$$

for quark and gluon-started splitting amplitude, respectively. So, roughly speaking, we are imposing 2 restrictions on $\mathcal{A}_{(\mathrm{amp}, g)}^{\mu}$ and just one on $\mathcal{A}_{(\mathrm{amp}, q)}^{i}$.

We would like to understand also which is the difference between gluon and photonstarted splitting functions. The last ones are extremely compact and, moreover, they do not 
involve integrals with LCG propagators. If we compare the Feynman diagrams required for $\gamma \rightarrow q \bar{q} \gamma$ and $g \rightarrow q \bar{q} \gamma$, we can see that non-Abelian interactions vanish completely in the first case. Moreover, changing the coupling constant and removing the global color factor, NLO QCD corrections to $\gamma \rightarrow q \bar{q} \gamma$ are exactly the same that the corresponding QED NLO corrections. Since QED is an Abelian theory, we can use a covariant gauge to compute virtual corrections and the result remains unchanged. However the same explanation can not be directly applied to $\gamma \rightarrow q \bar{q} g$ because of NA diagrams, although the central idea is related with gauge invariance. Note that splitting functions are computed using a fixed physical-gauge (LCG in particular) in order to simplify factorization properties in the collinear limit. If we restrict the analysis to the computational procedure, we find out that we are computing NLO QCD corrections to an amputated scattering amplitude with the incoming particle slightly off-shell, and then making a projection over an on-shell spinor/polarization vector. When the process is started by a QCD parton, there is a color flux across the off-shell parent leg. If the parent parton is a photon then

$$
\sum_{i=1}^{\bar{m}} \boldsymbol{T}^{i}=0,
$$

because photons are color singlets. This implies that we can attach the amputated amplitude to a pair of colorless fermions (for instance, an electron-positron pair) and reconstruct the full NLO QCD correction to the on-shell physical scattering amplitude. Since physical scattering amplitudes are gauge invariant, we can use covariant gauge and only standard scalar integrals are required in the computation. In other words, we can write

$$
\begin{aligned}
\left.\mathcal{A}_{e^{-} e^{+} \rightarrow a_{1} a_{2} a_{3}}^{(1)}\left(k_{1}, k_{2} ; p_{1}, p_{2}, p_{3}\right)\right|_{\mathrm{LCG}} & =\left.\bar{v}\left(k_{2}\right)\left(-\imath g_{e} \gamma^{\nu}\right) u\left(k_{1}\right) \frac{-\imath \eta_{\mu \nu}}{s_{123}} \mathcal{A}_{(\mathrm{amp}, \gamma)}^{(1, \mu)}\right|_{\mathrm{LCG}} \\
& =-g_{e} C_{\mathrm{pol}}\left[\left.\frac{1}{s_{123}} \frac{\bar{v}\left(k_{2}\right) \gamma_{\mu} u\left(k_{1}\right)}{C_{\mathrm{pol}}} \mathcal{A}_{(\mathrm{amp}, \gamma)}^{(1, \mu)}\right|_{\mathrm{LCG}}\right],
\end{aligned}
$$

where we are using physical momenta for the process $e^{-} e^{+} \rightarrow a_{1} a_{2} a_{3}\left(a_{i}\right.$ could be a QCD parton), which implies that $k_{i}^{2}=0$ (massless fermions on-shell) and $k_{1}+k_{2}=p_{1}+p_{2}+p_{3}$ (vector equation). Here $C_{\text {pol }}$ is an arbitrary normalization factor which only depends on the kinematics. Due to gauge invariance,

$$
\mathcal{A}_{e^{-} e^{+} \rightarrow a_{1} a_{2} a_{3}}^{(1)}\left(k_{1}, k_{2} ; p_{1}, p_{2}, p_{3}\right)=\left.\mathcal{A}_{e^{-} e^{+} \rightarrow a_{1} a_{2} a_{3}}^{(1)}\left(k_{1}, k_{2} ; p_{1}, p_{2}, p_{3}\right)\right|_{\mathrm{LCG}},
$$

which implies

$$
\left.\frac{\bar{v}\left(k_{2}\right) \gamma_{\mu} u\left(k_{1}\right)}{C_{\mathrm{pol}}} \mathcal{A}_{(\mathrm{amp}, \gamma)}^{(1, \mu)}\right|_{\mathrm{LCG}}=\frac{\bar{v}\left(k_{2}\right) \gamma_{\mu} u\left(k_{1}\right)}{C_{\mathrm{pol}}} \mathcal{A}_{(\mathrm{amp}, \gamma)}^{(1, \mu)} .
$$

This relation is true for every $k_{i}$ which fulfils physical conditions. In particular, we might use

$$
k_{1}^{\mu}=\widetilde{P}^{\mu}, \quad k_{2}^{\mu}=\frac{s_{123}}{2 n P} n^{\mu},
$$

or in the inverted order. However, if we restrict external polarizations to be physical and use one of the previously mentioned choices, then

$$
\frac{\bar{v}\left(k_{2}\right) \gamma_{\mu} u\left(k_{1}\right)}{C_{\mathrm{pol}}}=\epsilon_{\mu}^{ \pm}(\widetilde{P}, n),
$$


where we are applying the well-known mapping of polarization vectors into spinor chains defined in the spinor-helicity formalism (for instance, see ref. [32]). So, relying in eq. (4.34) and these results, we conclude that it is possible to use covariant gauge in the computation of loop diagrams inside a photon-started splitting amplitude. And, for the triple collinear limit, this fact directly implies that we can make the replacement $d_{\mu \nu}(q, n) \rightarrow-\eta_{\mu \nu}$ inside the loop integrals associated with the Feynman diagrams presented in figures 5 and 6 . This proof can be generalized for splittings with more colored particles in the final state.

\section{Conclusions}

In this article we present the triple collinear splitting functions at NLO accuracy for processes which involve photons. Computations are performed using DREG to make IR/UV divergences explicit and we choose to work with CDR scheme in order to simplify the analytic treatment of the expressions. The results are organized in such a way that the divergent structure is exposed explicitly. Moreover, this structure completely agrees with the predicted behavior according to Catani's formula. Besides that, the classification of terms according to their transcendental weight and exchange symmetries allows us to obtain very compact results. These splitting functions are computed in the time-like region (TL) where strict collinear factorization is fulfilled [2].

We have considered processes started by QCD partons and also by photons. In particular, we used these results to explore the possibility of crossing-like relations among splitting functions beyond LO. Since the parent parton is off-shell, crossing-symmetry is broken and it is not possible to relate splittings with the same particle content. The situation is different for the double collinear limit at LO because it involves a single energy scale. Anyway, even for double-collinear configurations, the study of splittings started by photons reveals that it is not possible to establish such a connection in the context of higher-order corrections in QCD+QED.

Besides providing explicit results for all triple-splitting functions with at least one photon, we explore the simplifications that occur in photon-started processes. We showed that results can be expressed in terms of standard scalar boxes and bubbles. Having all QCD partons on-shell imposes additional constraints which force the cancellation of LCG denominators inside loops. We give a proof of this fact based in the spinor-helicity formalism and gauge invariance.

Finally, we would like to discuss about the importance of higher orders in the $\epsilon$ expansion of the triple collinear splitting functions. One of the main motivations to compute these objects is related with $\mathrm{N}^{k} \mathrm{LO}$ corrections to physical cross-sections. The counterterms involved in subtraction-like methods require the convolution of splitting functions with some factors which contain $\epsilon$-poles. For example, at NLO, the typical form of the initial-state collinear counter-term is $[6,33]$

$$
d \sigma_{a_{1} a_{2} \rightarrow X}^{\mathrm{cnt}}=\frac{\alpha_{\mathrm{S}}}{2 \pi} \sum_{b} \int d z \frac{1}{\epsilon}\left\langle\hat{P}_{a_{1} \rightarrow b P\left(a_{1}, b\right)}^{(0)}(z, \epsilon)\right\rangle d \sigma_{b a_{2} \rightarrow X^{\prime}}^{(0)}
$$


which contains $\mathcal{O}\left(\epsilon^{0}\right)$ contributions arising from $\mathcal{O}(\epsilon)$ terms in the splitting functions. The codes that we employ to obtain the results shown in this article can be extended to compute $\mathcal{O}(\epsilon)$ terms (or even higher orders), although explicit analytic expressions might be extremely lengthy. Anyway, numerical results for the counter-terms could be calculated provided that we know the corresponding $\epsilon$-expansion of the involved master integrals.

\section{Acknowledgments}

We would like to thank Stefano Catani for making useful comments in an early stage of this project. Also, we would like to thanks M. V. Garzelli for the fruitful discussions about the implementation of numerical checks. This work is partially supported by UBACYT, CONICET, ANPCyT, the Research Executive Agency (REA) of the European Union under the Grant Agreement number PITN-GA-2010-264564 (LHCPhenoNet), by the Spanish Government and EU ERDF funds (grants FPA2011-23778 and CSD2007-00042 Consolider Ingenio CPAN) and by GV (PROMETEUII/2013/007).

Open Access. This article is distributed under the terms of the Creative Commons Attribution License (CC-BY 4.0), which permits any use, distribution and reproduction in any medium, provided the original author(s) and source are credited.

\section{References}

[1] J.C. Collins, D.E. Soper and G.F. Sterman, Factorization of hard processes in $Q C D$, in Perturbative quantum chromodynamics, A.H. Mueller ed., Adv. Ser. Direct. High Energy Phys. 5 (1988) 1 [hep-ph/0409313] [INSPIRE].

[2] S. Catani, D. de Florian and G. Rodrigo, Space-like (versus time-like) collinear limits in QCD: is factorization violated?, JHEP 07 (2012) 026 [arXiv: 1112.4405] [INSPIRE].

[3] F.A. Berends and W.T. Giele, Recursive calculations for processes with $N$ gluons, Nucl. Phys. B 306 (1988) 759 [inSPIRE].

[4] M.L. Mangano and S.J. Parke, Multiparton amplitudes in gauge theories, Phys. Rept. 200 (1991) 301 [hep-th/0509223] [INSPIRE].

[5] G. Altarelli and G. Parisi, Asymptotic freedom in parton language, Nucl. Phys. B 126 (1977) 298 [INSPIRE].

[6] S. Catani and M.H. Seymour, A general algorithm for calculating jet cross-sections in NLO QCD, Nucl. Phys. B 485 (1997) 291 [Erratum ibid. B 510 (1998) 503] [hep-ph/9605323] [INSPIRE].

[7] Z. Bern, V. Del Duca and C.R. Schmidt, The infrared behavior of one loop gluon amplitudes at next-to-next-to-leading order, Phys. Lett. B 445 (1998) 168 [hep-ph/9810409] [INSPIRE].

[8] Z. Bern, V. Del Duca, W.B. Kilgore and C.R. Schmidt, The infrared behavior of one loop QCD amplitudes at next-to-next-to leading order, Phys. Rev. D 60 (1999) 116001 [hep-ph/9903516] [INSPIRE].

[9] Z. Bern, G. Chalmers, L.J. Dixon and D.A. Kosower, One loop $N$ gluon amplitudes with maximal helicity violation via collinear limits, Phys. Rev. Lett. 72 (1994) 2134 [hep-ph/9312333] [INSPIRE]. 
[10] Z. Bern, L.J. Dixon, D.C. Dunbar and D.A. Kosower, One loop $N$ point gauge theory amplitudes, unitarity and collinear limits, Nucl. Phys. B 425 (1994) 217 [hep-ph/9403226] [INSPIRE].

[11] Z. Bern and G. Chalmers, Factorization in one loop gauge theory, Nucl. Phys. B 447 (1995) 465 [hep-ph/9503236] [INSPIRE].

[12] D.A. Kosower and P. Uwer, One loop splitting amplitudes in gauge theory, Nucl. Phys. B 563 (1999) 477 [hep-ph/9903515] [INSPIRE].

[13] Z. Bern, L.J. Dixon and D.A. Kosower, Two-loop $g \rightarrow$ gg splitting amplitudes in QCD, JHEP 08 (2004) 012 [hep-ph/0404293] [INSPIRE].

[14] S.D. Badger and E.W.N. Glover, Two loop splitting functions in QCD, JHEP 07 (2004) 040 [hep-ph/0405236] [INSPIRE].

[15] A. Vogt, S. Moch and J. Vermaseren, Photon-parton splitting functions at the next-to-next-to-leading order of QCD, Acta Phys. Polon. B 37 (2006) 683 [hep-ph/0511112] [INSPIRE].

[16] A. Vogt, S. Moch and J.A.M. Vermaseren, The three-loop splitting functions in QCD: the singlet case, Nucl. Phys. B 691 (2004) 129 [hep-ph/0404111] [INSPIRE].

[17] S. Moch, J.A.M. Vermaseren and A. Vogt, The three loop splitting functions in QCD: the nonsinglet case, Nucl. Phys. B 688 (2004) 101 [hep-ph/0403192] [INSPIRE].

[18] J.M. Campbell and E.W.N. Glover, Double unresolved approximations to multiparton scattering amplitudes, Nucl. Phys. B 527 (1998) 264 [hep-ph/9710255] [INSPIRE].

[19] S. Catani and M. Grazzini, Collinear factorization and splitting functions for next-to-next-to-leading order QCD calculations, Phys. Lett. B 446 (1999) 143 [hep-ph/9810389] [INSPIRE].

[20] V. Del Duca, A. Frizzo and F. Maltoni, Factorization of tree QCD amplitudes in the high-energy limit and in the collinear limit, Nucl. Phys. B 568 (2000) 211 [hep-ph/9909464] [INSPIRE].

[21] T.G. Birthwright, E.W.N. Glover, V.V. Khoze and P. Marquard, Multi-gluon collinear limits from MHV diagrams, JHEP 05 (2005) 013 [hep-ph/0503063] [INSPIRE].

[22] T.G. Birthwright, E.W.N. Glover, V.V. Khoze and P. Marquard, Collinear limits in $Q C D$ from MHV rules, JHEP 07 (2005) 068 [hep-ph/0505219] [INSPIRE].

[23] S. Catani and M. Grazzini, Infrared factorization of tree level QCD amplitudes at the next-to-next-to-leading order and beyond, Nucl. Phys. B 570 (2000) 287 [hep-ph/9908523] [INSPIRE].

[24] S. Catani, D. de Florian and G. Rodrigo, The triple collinear limit of one loop QCD amplitudes, Phys. Lett. B 586 (2004) 323 [hep-ph/0312067] [INSPIRE].

[25] C.G. Bollini and J.J. Giambiagi, Dimensional renormalization: the number of dimensions as a regularizing parameter, Nuovo Cim. 12 (1972) 20 [INSPIRE].

[26] G. 't Hooft and M.J.G. Veltman, Regularization and renormalization of gauge fields, Nucl. Phys. B 44 (1972) 189 [INSPIRE].

[27] Z. Kunszt, A. Signer and Z. Trócsányi, One loop helicity amplitudes for all $2 \rightarrow 2$ processes in $Q C D$ and $N=1$ supersymmetric Yang-Mills theory, Nucl. Phys. B 411 (1994) 397 [hep-ph/9305239] [INSPIRE]. 
[28] P. Draggiotis, M.V. Garzelli, C.G. Papadopoulos and R. Pittau, Feynman rules for the rational part of the QCD 1-loop amplitudes, JHEP 04 (2009) 072 [arXiv:0903.0356] [INSPIRE].

[29] M.V. Garzelli, I. Malamos and R. Pittau, Feynman rules for the rational part of the electroweak 1-loop amplitudes, JHEP 01 (2010) 040 [Erratum ibid. 10 (2010) 097] [arXiv:0910.3130] [INSPIRE].

[30] G.F.R. Sborlini, D. de Florian and G. Rodrigo, Double collinear splitting amplitudes at next-to-leading order, JHEP 01 (2014) 018 [arXiv:1310.6841] [INSPIRE].

[31] J.R. Forshaw, M.H. Seymour and A. Siodmok, On the breaking of collinear factorization in QCD, JHEP 11 (2012) 066 [arXiv:1206.6363] [INSPIRE].

[32] L.J. Dixon, Calculating scattering amplitudes efficiently, in $Q C D$ and beyond, Boulder U.S.A. (1995), pg. 539 [hep-ph/9601359] [INSPIRE].

[33] S. Frixione, Z. Kunszt and A. Signer, Three jet cross-sections to next-to-leading order, Nucl. Phys. B 467 (1996) 399 [hep-ph/9512328] [INSPIRE]. 